\title{
Burst synchronization in a scale-free neuronal network with inhibitory spike-timing-dependent plasticity
}

\author{
Sang-Yoon $\mathrm{Kim}^{1} \cdot$ Woochang Lim $^{1}$
}

Received: 9 May 2018/Revised: 19 August 2018/Accepted: 28 August 2018/Published online: 11 September 2018

(C) Springer Nature B.V. 2018

\begin{abstract}
We are concerned about burst synchronization (BS), related to neural information processes in health and disease, in the Barabási-Albert scale-free network (SFN) composed of inhibitory bursting Hindmarsh-Rose neurons. This inhibitory neuronal population has adaptive dynamic synaptic strengths governed by the inhibitory spike-timing-dependent plasticity (iSTDP). In previous works without considering iSTDP, BS was found to appear in a range of noise intensities for fixed synaptic inhibition strengths. In contrast, in our present work, we take into consideration iSTDP and investigate its effect on BS by varying the noise intensity. Our new main result is to find occurrence of a Matthew effect in inhibitory synaptic plasticity: good BS gets better via LTD, while bad BS get worse via LTP. This kind of Matthew effect in inhibitory synaptic plasticity is in contrast to that in excitatory synaptic plasticity where good (bad) synchronization gets better (worse) via LTP (LTD). We note that, due to inhibition, the roles of LTD and LTP in inhibitory synaptic plasticity are reversed in comparison with those in excitatory synaptic plasticity. Moreover, emergences of LTD and LTP of synaptic inhibition strengths are intensively investigated via a microscopic method based on the distributions of time delays between the pre- and the post-synaptic burst onset times. Finally, in the presence of iSTDP we investigate the effects of network architecture on BS by varying the symmetric attachment degree $l^{*}$ and the asymmetry parameter $\Delta l$ in the SFN.
\end{abstract}

Keywords Inhibitory spike-timing-dependent plasticity $\cdot$ Burst synchronization $\cdot$ Scale-free network $\cdot$ Bursting neurons

\section{Introduction}

Recently, population synchronization of bursting neurons has attracted much attention in many aspects (Elson et al. 1998; Stern et al. 1998; Varona et al. 2001; van Vreeswijk and Hansel 2001; Dhamala et al. 2004; Ivanchenko et al. 2004; Shilnikov and Cymbalyuk 2005; Shi and Lu 2005, 2009; Tanaka et al. 2006; Pereira et al. 2007; Batista et al. 2007, 2009, 2010, 2012; Lameu et al. 2012; Wang et al. 2009, 2011a, b, 2013; Sun et al. 2011; Yu et al. 2011; Duan et al. 2013; Meng et al. 2013; Hrg 2013; Prado et al. 2014; Ferrari et al. 2015; Ngouonkadi et al. 2016; Rostami

Woochang Lim

wclim@icn.re.kr

Sang-Yoon Kim

sykim@icn.re.kr

1 Institute for Computational Neuroscience and Department of Science Education, Daegu National University of Education, Daegu 42411, Korea and Jafari 2018). There are several representative examples of bursting neurons such as intrinsically bursting neurons and chattering neurons in the cortex (Connors and Gutnick 1990; Gray and McCormick 1996), thalamic relay neurons and thalamic reticular neurons in the thalamus (Llinás and Jahnsen 1982; McCormick and Huguenard 1992; Lee et al. 2007), hippocampal pyramidal neurons (Su et al. 2001), Purkinje cells in the cerebellum (Womack and Khodakhah 2002), pancreatic $\beta$-cells (Chay and Keizer 1983; Pernarowski et al. 1992; Kinard et al. 1999), and respiratory neurons in pre-Botzinger complex (Del Negro et al. 1998; Butera et al. 1999). As is well known, burstings occur when neuronal activity alternates, on a slow timescale, between a silent phase and an active (bursting) phase of fast repetitive spikings (Rinzel 1985, 1987; Izhikevich 2000; Coombes and Bressloff 2005; Izhikevich 2006, 2007). Due to a repeated sequence of spikes in the bursting, there are many hypotheses on the importance of bursting activities in neural computation (Lisman 1997; Izhikevich et al. 2003; Krahe and Gabbian 2004; 
Izhikevich 2004, 2006); for example, (a) bursts are necessary to overcome the synaptic transmission failure, (b) bursts are more reliable than single spikes in evoking responses in post-synaptic neurons, (c) bursts evoke longterm potentiation/depression (and hence affect synaptic plasticity much greater than single spikes), and (d) bursts can be used for selective communication between neurons.

Here, we are interested in burst synchronization (BS) (i.e., synchrony on the slow bursting timescale) which characterizes temporal coherence between the (active phase) burst onset times (i.e., times at which burstings start in active phases). This type of BS is associated with neural information processes in health and disease. For example, large-scale BS (called the sleep spindle oscillation of $7-14 \mathrm{~Hz}$ ) has been found to occur through interaction between the excitatory thalamic relay cells and the inhibitory thalamic reticular neurons in the thalamus during the early stage of slow-wave sleep (Steriade et al. 1993; Bazhenov and Timofeev 2006). These sleep spindle oscillations are involved in memory consolidation (Gais et al. 2000; Sejnowski and Destexhe 2000). On the other hand, BS is also correlated to abnormal pathological rhythms, associated with neural diseases such as movement disorder (Parkinson's disease and essential tremor) (Bevan et al. 2002; Uhlhaas and Singer 2006; Brown 2007; Hammond et al. 2007; Park et al. 2010) and epileptic seizure (Fisher et al. 2005; Uhlhaas and Singer 2006). For the case of the Parkinson's disease hypokinetic motor symptoms (i.e., slowness and rigidity of voluntary movement) are closely associated with BS occurring in the beta band of $10-30 \mathrm{~Hz}$ range in the basal ganglia, while the hyperkinetic motor symptom (i.e., resting tremor) is related to BS in the theta band of $3-10 \mathrm{~Hz}$.

In real brains, synaptic strengths may change for adaptation to the environment [i.e., they can be potentiated (Kornoski 1948; Hebb 1949; Shatz 1992) or depressed (Stent 1973; von der Malsburg 1973; Sejnowski 1977; Bienenstock et al. 1982)]. These adjustments of synapses are called the synaptic plasticity which provides the basis for learning, memory, and development (Abbott and Nelson 2000). Such synaptic plasticity is taken into consideration in the present work. As to the synaptic plasticity, we consider a spike-timing-dependent plasticity (STDP) (Song et al. 2000; Bi and Poo 2001; Kepecs et al. 2002; Dan and Poo 2004, 2006; Caporale and Dan 2008; Feldman 2012; Markram et al. 2012). For the STDP, the synaptic strengths vary via an update rule depending on the relative time difference between the pre- and the post-synaptic burst onset times. Many models for STDP have been employed to explain results on synaptic modifications occurring in diverse neuroscience topics for health and disease such as temporal sequence learning (Abbott and Blum 1996), temporal pattern recognition (Feldman 2000), coincidence detection (Gerstner et al. 1996), navigation (Blum and Abbott 1996), direction selectivity (Mehta and Wilson 2000), memory consolidation (Ji and Wilson 2007), competitive/selective development (Song and Abbott 2001), and deep brain stimulation (Lourens et al. 2015; Grado et al. 2015). Recently, the effects of STDP on population synchronization in ensembles of coupled neurons were also studied in various aspects (Popovych and Tass 2012; Popovych et al. 2013; Borges et al. 2016, 2017a; Kim and Lim 2018a, b, c, d).

Here, we study emergence of BS in a scale-free network (SFN) of inhibitory bursting neurons. In the absence of synaptic plasticity (i.e., coupling strengths are static), BS has been found to appear in a range of noise intensity $D$ for a fixed coupling strength (Kim and Lim 2016). As $D$ is increased from 0 , the degree of BS decreases due to a destructive role of noise to spoil the BS, and when passing a threshold a transition from BS to desynchronization occurs. In contrast to the previous work, we now take into consideration the synaptic plasticity, and then the inhibitory population has adaptive dynamic synaptic strengths governed by the inhibitory spike-timing-dependent plasticity (iSTDP). Studies of synaptic plasticity have been mainly focused on excitatory-to-excitatory (E-to-E) synaptic connections between excitatory pyramidal cells (Gerstner et al. 1996; Markram et al. 1997; Bi and Poo 1998; Debanne et al. 1998; Zhang et al. 1998; Egger et al. 1999; Feldman 2000; Tzounopoulos et al. 2004; Wittenberg and Wang 2006). An asymmetric Hebbian time window was employed for the excitatory STDP (eSTDP) update rule (Song et al. 2000; Bi and Poo 2001; Kepecs et al. 2002; Dan and Poo 2004, 2006; Caporale and Dan 2008; Feldman 2012; Markram et al. 2012). When a presynaptic spiking precedes a post-synaptic spiking, longterm potentiation (LTP) occurs; otherwise, long-term depression (LTD) appears. On the other hand, plasticity of inhibitory connections has attracted less attention mainly due to experimental obstacles and diversity of inhibitory interneurons (Gaiarsa et al. 2002; Lamsa et al. 2010; Kullmann et al. 2012; Vogels et al. 2013; Froemke 2015). Along with the advent of fluorescent labeling and optical manipulation of neurons according to their genetic types (Deisseroth et al. 2006; Cardin 2012), inhibitory plasticity has also begun to be focused. Particularly, studies on iSTDP of inhibitory-to-excitatory (I to E) connections have been made. Thus, iSTDP has been found to be diverse and cell-specific (Soto-Trevino et al. 2001; Gaiarsa et al. 2002; Woodin et al. 2003; Haas et al. 2006; Talathi et al. 2008; Lamsa et al. 2010; Vogels et al. 2011, 2013; Castilo et al. 2011; Kullmann et al. 2012; Froemke 2015; Borges et al. 2017b).

In this paper, we consider an inhibitory Barabási-Albert SFN consisting of bursting neurons (Barabási and Albert 
1999; Albert and Barabási 2002), and investigate the effect of iSTDP [of inhibitory-to-inhibitory (I to I) connections] on $\mathrm{BS}$ by varying the noise intensity $D$. As mentioned above, previous studies on iSTDP have been focused mainly on the case of inhibitory-to-excitatory (I to E) connections. Even in this case of I to E iSTDP, time windows for the iSTDP rule vary depending on the target pyramidal cells [e.g., delayed Hebbian time window (Haas et al. 2006; Talathi et al. 2008) for the pyramidal cells in the entorhinal cortex and symmetric time window (Woodin et al. 2003) for the pyramidal cells in the CA1 hippocampus]. In our present work, we consider the I to I iSTDP; the target neuron is inhibitory, in contrast to the above excitatory case. Recently, such I to I iSTDP has been studied in works (Popovych and Tass 2012; Lourens et al. 2015; Grado et al. 2015) where the anti-Hebbian time window was used for the I to I iSTDP. Following them, we also employ the anti-Hebbian time window for studying the effect of I to I iSTDP on BS. As the time is increased, strengths of synaptic inhibition $\left\{J_{i j}\right\}$ are changed, and eventually, they approach saturated limit values after a sufficiently long time. Depending on $D$, mean values of saturated synaptic inhibition strengths $\left\{J_{i j}^{*}\right\}$ are potentiated [long-term potentiation (LTP)] or depressed [long-term depression (LTD)], in comparison with the initial mean value of synaptic inhibition strengths. In contrast, standard deviations from the mean values of LTP/LTD are much increased, when compared with the initial dispersion, independently of $D$. Both the mean value and the standard deviation (for the distribution of synaptic inhibition strengths) may affect BS. The LTD (LTP) tends to increase (decrease) the degree of BS due to decrease (increase) in the mean value of synaptic inhibition strengths, and the increased standard deviations have a tendency to decrease the degree of BS. In most range of $D$ with LTD, good BS (with higher bursting measure) gets better because the effect of mean LTD is dominant in comparison with the effect of increased standard deviation. In contrast, in the range of $D$ with LTP, bad BS (with lower bursting measure) gets worse due to the effects of both LTP and increased standard deviation. We note that this effect is similar to the Matthew effect in the sociology of science (Merton 1968); the rich get richer and the poor get poorer. Hence, for our case, we call the effect of iSTDP on BS as the Matthew effect in inhibitory synaptic plasticity (Kim and Lim 2018d), in addition to the Matthew effect in excitatory synaptic plasticity in previous works (Kim and Lim 2018a, b, c). This type of Matthew effect in inhibitory synaptic plasticity is in contrast to that in excitatory synaptic plasticity where good (bad) synchronization gets better (worse) via LTP (LTD) (Kim and Lim 2018a, b, c). We note that the role of LTD (LTP) in the case of iSTDP is similar to that of LTP (LTD) for the case of eSTDP. Emergences of LTD and LTP of synaptic inhibition strengths are also investigated through a microscopic method based on the distributions of time delays between the nearest burst onset times of the pre- and the post-synaptic neurons. Furthermore, in the presence of iSTDP we study the effects of network architecture on BS for a fixed value of $D$ by varying the symmetric attachment degree $l^{*}$ and the asymmetry parameter $\Delta l$ in the SFN. Like the above case of variation in $D$, Matthew effects in inhibitory synaptic plasticity are also found to occur by varying $l^{*}$ and $\Delta l$.

For the sake of clearness, we also make a brief summary of new main results in our present work. In a previous work (Kim and Lim 2016), BS was found to occur in a range of noise intensities for static synaptic inhibition strengths. However, in real brains, synaptic strengths may change for adaptation to the environment (i.e., they may be potentiated or depressed); these adjustments of synapses are called the synaptic plasticity. In our present work, we take into consideration the iSTDP and investigate its effect on BS by varying the noise intensity, in contrast to the previous works without considering iSTDP. Our new main finding is occurrence of the Matthew effect in inhibitory synaptic plasticity: good BS gets better via LTD, while bad BS get worse via LTP. This type of Matthew effect in inhibitory synaptic plasticity is in contrast to the Matthew effect in excitatory synaptic plasticity (Kim and Lim 2018a, b, c) where good (bad) synchronization gets better (worse) via LTP (LTD). We note that, due to inhibition, the roles of LTD and LTP in inhibitory synaptic plasticity are reversed in comparison with those in excitatory synaptic plasticity. Furthermore, our results on the effect of iSTDP on BS are also expected to be useful for understanding the basis for not only the fundamental brain function (e.g., learning, memory, and development) (Abbott and Blum 1996; Ji and Wilson 2007; Song and Abbott 2001), but also neural diseases (e.g., Parkinsons disease and epilepsy) (Bevan et al. 2002; Fisher et al. 2005; Uhlhaas and Singer 2006; Brown 2007; Hammond et al. 2007; Park et al. 2010).

This paper is organized as follows. In "Scale-free network of inhibitory bursting Hindmarsh-Rose neurons" section, we describe an inhibitory Barabási-Albert SFN of bursting neurons with inhibitory synaptic plasticity. Then, in "Effects of inhibitory STDP on burst synchronization" section we investigate the effects of iSTDP on BS. Finally, a summary is given in "Summary" section. 


\section{Scale-free network of inhibitory bursting Hindmarsh-Rose neurons}

Synaptic connectivity in brain networks has been found to have complex topology which is neither regular nor completely random (Sporns et al. 2000; Buzsáki et al. 2004; Chklovskii et al. 2004; Song et al. 2005; Bassett and Bullmore 2006; Sporns and Honey 2006; Larimer and Strowbridge 2008; Bullmore and Sporns 2009; Sporns 2011). Particularly, brain networks have been found to exhibit power-law degree distributions (i.e., scale-free property) in the rat hippocampal networks (Morgan and Soltesz 2008; Bonifazi et al. 2009; Wiedemann 2010; Li et al. 2010) and the human cortical functional network (Eguíluz et al. 2005). Furthermore, robustness against simulated lesions of mammalian cortical anatomical networks (Felleman and Van Essen 1991; Young 1993; Young et al. 1994; Scannell et al. 1995, 1999; Sporns et al. 2004) has also been found to be most similar to that of an SFN (Kaiser et al. 2007). Many recent works on various subjects of neurodynamics (e.g., coupling-induced BS, delay-induced BS, and suppression of BS) have been done in SFNs with a few percent of hub neurons with an exceptionally large number of connections (Batista et al. 2007, 2009, 2010; Wang et al. 2009, 2011b; Ferrari et al. 2015).

We consider an inhibitory SFN composed of $N$ bursting neurons equidistantly placed on a one-dimensional ring of radius $N / 2 \pi$. We employ a directed Barabási-Albert SFN model (i.e. growth and preferential directed attachment) (Barabási and Albert 1999; Albert and Barabási 2002). At each discrete time $t$, a new node is added, and it has $l_{\text {in }}$ incoming (afferent) edges and $l_{\text {out }}$ outgoing (efferent) edges via preferential attachments with $l_{\text {in }}$ (pre-existing) source nodes and $l_{\text {out }}$ (pre-existing) target nodes, respectively. The (pre-existing) source and target nodes $i$ (which are connected to the new node) are preferentially chosen depending on their out-degrees $d_{i}^{(\text {out })}$ and in-degrees $d_{i}^{(i n)}$ according to the attachment probabilities $\Pi_{\text {source }}\left(d_{i}^{(\text {out })}\right)$ and $\Pi_{\text {target }}\left(d_{i}^{(i n)}\right)$, respectively:

$$
\begin{aligned}
\Pi_{\text {source }}\left(d_{i}^{(\text {out })}\right) & =\frac{d_{i}^{(\text {out })}}{\sum_{j=1}^{N_{t-1}} d_{j}^{(\text {out })}} \text { and } \\
\Pi_{\text {target }}\left(d_{i}^{(\text {in })}\right) & =\frac{d_{i}^{(\text {in })}}{\sum_{j=1}^{N_{t-1}} d_{j}^{(\text {in })}},
\end{aligned}
$$

where $N_{t-1}$ is the number of nodes at the time step $t-1$. Hereafter, the cases of $l_{\text {in }}=l_{\text {out }}\left(\equiv l^{*}\right)$ and $l_{\text {in }} \neq l_{\text {out }}$ will be referred to as symmetric and asymmetric preferential attachments, respectively. For generation of an SFN with $N$ nodes, we begin with the initial network at $t=0$, consisting of $N_{0}=50$ nodes where the node 1 is connected bidirectionally to all the other nodes, but the remaining nodes (except the node 1) are sparsely and randomly connected with a low probability $p=0.1$. The processes of growth and preferential attachment are repeated until the total number of nodes becomes $N$. For this case, the node 1 will be grown as the head hub with the highest degree. Then, the grown network via the above process becomes scale free, because the distributions for the in- and the outdegrees $d^{(i n)}$ and $d^{(o u t)}$ exhibit power-law decays with the same exponent $\quad \gamma=3, \quad P\left(d^{(i n)}\right) \sim d^{(i n)^{-\gamma}} \quad$ and $P\left(d^{(\text {out })}\right) \sim d^{(\text {out })^{-\gamma}}$ (Barabási and Albert 1999; Albert and Barabási 2002).

As an element in our SFN, we choose the representative bursting HR neuron model which was originally introduced to describe the time evolution of the membrane potential for the pond snails (Hindmarsh and Rose 1982, 1984; Rose and Hindmarsh 1985); this HR neuron model was studied in many aspects (Duan et al. 2013; Hrg 2013; Wang et al. 2013; Jun et al. 2014; Kim and Lim 2015a, b, 2016; Ngouonkadi et al. 2016; Rostami and Jafari 2018; Zhu and Liu 2018). We consider the Barabási-Albert SFN composed of $N$ HR bursting neurons. The following Eqs. (2)(4) govern the population dynamics in the SFN:

$$
\begin{aligned}
& \frac{d x_{i}}{d t}=y_{i}-a x_{i}^{3}+b x_{i}^{2}-z_{i}+I_{D C, i}+D \xi_{i}-I_{s y n, i}, \\
& \frac{d y_{i}}{d t}=c-d x_{i}^{2}-y_{i}, \\
& \frac{d z_{i}}{d t}=r\left[s\left(x_{i}-x_{o}\right)-z_{i}\right],
\end{aligned}
$$

where

$$
\begin{aligned}
I_{s y n, i} & =\frac{1}{d_{i}^{(i n)}} \sum_{j=1(j \neq i)}^{N} J_{i j} w_{i j} g_{j}(t)\left(x_{i}-X_{s y n}\right), \\
g_{j}(t) & =\sum_{f=1}^{F_{j}} E\left(t-t_{f}^{(j)}-\tau_{l}\right) ; \\
E(t) & =\frac{1}{\tau_{d}-\tau_{r}}\left(e^{-t / \tau_{d}}-e^{-t / \tau_{r}}\right) \Theta(t) .
\end{aligned}
$$

Here, the state of the $i$ th neuron at a time $t$ (measured in units of milliseconds) is characterized by three state variables: the fast membrane potential $x_{i}$, the fast recovery current $y_{i}$, and the slow adaptation current $z_{i}$. The parameter values used in our computations are listed in Table 1. More details on external stimulus on the single HR neuron, synaptic currents and synaptic plasticity, and numerical integration of the governing equations are given in the following subsections. 
Table 1 Parameter values used in our computations
(1)

(2)

(5)
Single HR bursting neurons (Longtin 1997)

$a=1 \quad b=3 \quad c=1 \quad d=5 \quad r=0.001 \quad s=4 \quad x_{0}=-1.6$

External stimulus to HR bursting neurons

$I_{D C, i} \in[1.3,1.4] \quad D$ : Varying

Inhibitory synapse mediated by the $\mathrm{GABA}_{\mathrm{A}}$ neurotransmitter (Brunel and Wang 2003)

$\tau_{l}=1 \quad \tau_{r}=0.5 \quad \tau_{d}=5 \quad X_{\text {syn }}=-2$

Synaptic connections between neurons in the Barabási-Albert SFN

$l^{*}$ : Varying (symmetric preferential attachment)

$\Delta l$ : Varying (asymmetric preferential attachment)

$J_{0}=12 \quad \sigma_{0}=0.1 \quad J_{i j} \in[0.0001,20]$

Anti-Hebbian STDP rule

$\delta=0.08 \quad A_{+}=1.0 \quad A_{-}=1.3 \quad \tau_{+}=410 \quad \tau_{-}=330$

\section{External stimulus to each HR neuron}

Each bursting HR neuron [whose parameter values are in the 1st item of Table 1 (Longtin 1997)] is stimulated by a DC current $I_{D C, i}$ and an independent Gaussian white noise $\xi_{i}$ [see the 5th and the 6th terms in Eq. (2)] satisfying $\left\langle\xi_{i}(t)\right\rangle=0$ and $\left\langle\xi_{i}(t) \xi_{j}\left(t^{\prime}\right)\right\rangle=\delta_{i j} \delta\left(t-t^{\prime}\right)$, where $\langle\cdots\rangle$ denotes the ensemble average. The intensity of noise $\xi_{i}$ is controlled by the parameter $D$. Figure 1a shows a resting state of a single HR neuron for $I_{D C}=1.23$ in the absence of noise (i.e., $D=0$ ). As $I_{D C}$ passes a threshold $I_{D C}^{*}(\simeq 1.26)$, each single HR neuron exhibits a transition from a resting state to a bursting state. For the suprathreshold case of $I_{D C}=1.35$, deterministic bursting occurs when neuronal activity alternates, on a slow time scale $(\simeq 578 \mathrm{~ms}$ ), between a silent phase and an active (bursting) phase of fast repetitive spikings, as shown in Fig. 1b. The dotted horizontal line $\left(x_{b}^{*}=-1\right)$ denotes the bursting threshold (the solid and open circles denote the active phase onset and offset times, respectively), while the dashed horizontal line $\left(x_{s}^{*}=0\right)$ represents the spiking threshold within the active phase. An active phase of the bursting activity begins (ends) at a burst onset (offset) time when the membrane potential $x$ of the bursting HR neuron passes the bursting threshold of $x_{b}^{*}=-1$ from below
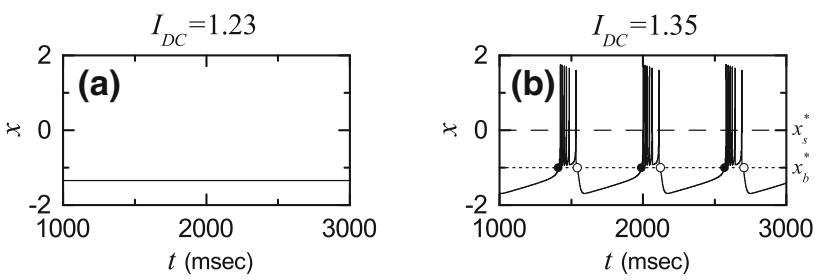

Fig. 1 Single bursting HR neuron for $D=0$. a Time series of $x(t)$ for the resting state when $I_{D C}=1.23$. b Time series of $x(t)$ for the bursting state when $I_{D C}=1.35$. The dotted horizontal line $\left(x_{b}^{*}=-1\right)$ and the dashed horizontal line $\left(x_{s}^{*}=0\right)$ represent the bursting and spiking thresholds, respectively. The solid and open circles denote the burst onset and offset times, respectively (above). For this case, the HR neuron exhibits bursting activity with the slow bursting frequency $f_{b}(\simeq 1.7 \mathrm{~Hz})$ [corresponding to the average inter-burst interval (IBI) ( $\simeq 578 \mathrm{~ms}$ )]. Throughout this paper, we consider a suprathreshold case such that the value of $I_{D C, i}$ is chosen via uniform random sampling in the range of $[1.3,1.4]$, as shown in the 2 nd item of Table 1 .

\section{Synaptic currents and plasticity}

The last term in Eq. (2) represents the synaptic couplings of HR bursting neurons. $I_{s y n, i}$ of Eq. (5) represents a synaptic current injected into the $i$ th neuron, and $X_{s y n}$ is the synaptic reversal potential. The synaptic connectivity is given by the connection weight matrix $W\left(=\left\{w_{i j}\right\}\right)$ where $w_{i j}=1$ if the bursting neuron $j$ is presynaptic to the bursting neuron $i$; otherwise, $w_{i j}=0$. Here, the synaptic connection is modeled in terms of the Barabási-Albert SFN. Then, the in-degree of the $i$ th neuron, $d_{i}^{(i n)}$ (i.e., the number of synaptic inputs to the interneuron $i$ ) is given by $d_{i}^{(i n)}=\sum_{j=1(j \neq i)}^{N} w_{i j}$. The fraction of open synaptic ion channels at time $t$ is denoted by $g(t)$. The time course of $g_{j}(t)$ of the $j$ th neuron is given by a sum of delayed doubleexponential functions $E\left(t-t_{f}^{(j)}-\tau_{l}\right)$ [see Eq. (6)], where $\tau_{l}$ is the synaptic delay, and $t_{f}^{(j)}$ and $F_{j}$ are the $f$ th spike and the total number of spikes of the $j$ th neuron at time $t$, respectively. Here, $E(t)$ [which corresponds to contribution of a presynaptic spike occurring at time 0 to $g_{j}(t)$ in the absence of synaptic delay] is controlled by the two synaptic time constants: synaptic rise time $\tau_{r}$ and decay time $\tau_{d}$, and $\Theta(t)$ is the Heaviside step function: $\Theta(t)=1$ for $t \geq 0$ and 0 for $t<0$. For the inhibitory GABAergic synapse (involving the $\mathrm{GABA}_{\mathrm{A}}$ receptors), the values of $\tau_{l}, \tau_{r}, \tau_{d}$, and $X_{\text {syn }}$ are listed in the 3rd item of Table 1 (Brunel and Wang 2003).

The coupling strength of the synapse from the $j$ th presynaptic neuron to the $i$ th post-synaptic neuron is $J_{i j}$. Here, 
we consider a multiplicative iSTDP (dependent on states) for the synaptic strengths $\left\{J_{i j}\right\}$ (Popovych et al. 2013; Rubin et al. 2001). To avoid unbounded growth and elimination of synaptic connections, we set a range with the upper and the lower bounds: $J_{i j} \in\left[J_{l}, J_{h}\right]$, where $J_{l}=$ 0.0001 and $J_{h}=20$. Initial synaptic strengths are normally distributed with the mean $J_{0}(=12)$ and the standard deviation $\sigma_{0}(=0.1)$. With increasing time $t$, the synaptic strength for each synapse is updated with a nearest-burst pair-based STDP rule (Morrison et al. 2007):

$J_{i j} \rightarrow J_{i j}+\delta\left(J^{*}-J_{i j}\right)\left|\Delta J_{i j}\left(\Delta t_{i j}\right)\right|$,

where $\delta(=0.08)$ is the update rate, $J^{*}=J_{h}\left(J_{l}\right)$ for the LTP (LTD), and $\Delta J_{i j}\left(\Delta t_{i j}\right)$ is the synaptic modification depending on the relative time difference $\Delta t_{i j}\left(=t_{i}^{(p o s t)}\right.$ $\left.t_{j}^{(\text {pre })}\right)$ between the nearest burst onset times of the postsynaptic neuron $i$ and the pre-synaptic neuron $j$. We use an asymmetric anti-Hebbian time window for the synaptic modification $\Delta J_{i j}\left(\Delta t_{i j}\right)$ (Popovych and Tass 2012; Lourens et al. 2015; Grado et al. 2015):

$\Delta J_{i j}\left(\Delta t_{i j}\right)=\left\{\begin{array}{ll}-A_{+} e^{-\Delta t_{i j} / \tau_{+}} & \text {for } \Delta t_{i j}>0 \\ -A_{-} \frac{\Delta t_{i j}}{\tau_{-}} e^{\Delta t_{i j} / \tau_{-}} & \text {for } \Delta t_{i j} \leq 0\end{array}\right.$,

where $\quad A_{+}=1.0, \quad A_{-}=1.3, \quad \tau_{+}=410 \mathrm{~ms}, \quad$ and $\tau_{-}=330 \mathrm{~ms}$ (these values are also given in the 5 th item of Table 1). Figure 2 shows an asymmetric anti-Hebbian time window for $\Delta J_{i j}\left(\Delta t_{i j}\right)$ of Eq. (8) (i.e., plot of $\Delta J_{i j}$ vs. $\Delta t_{i j}$ ). $\Delta J_{i j}\left(\Delta t_{i j}\right)$ changes depending on the relative time difference $\Delta t_{i j}\left(=t_{i}^{(\text {post })}-t_{j}^{(\text {pre })}\right)$ between the nearest burst onset times of the post-synaptic neuron $i$ and the pre-synaptic neuron $j$. In contrast to the case of a Hebbian time window for the eSTDP (Kim and Lim 2018a, b, c), when a postsynaptic burst follows a pre-synaptic burst (i.e., $\Delta t_{i j}$ is

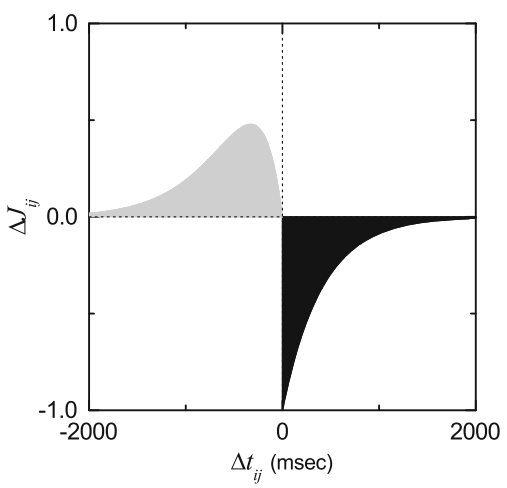

Fig. 2 Time window for the Anti-Hebbian iSTDP. Plot of synaptic modification $\Delta J_{i j}$ versus $\Delta t_{i j}\left(=t_{i}^{\text {post })}-t_{j}^{(\text {pre })}\right)$ for $A_{+}=1, A_{-}=1.3$, $\tau_{+}=410 \mathrm{~ms}$ and $\tau_{-}=330 \mathrm{~ms}$. $t_{i}^{(\text {post })}$ and $t_{j}^{(\text {pre })}$ are burst onset times of the $i$ th post-synaptic and the $j$ th pre-synaptic neurons, respectively positive), LTD of synaptic strength appears in the black region; otherwise (i.e., $\Delta t_{i j}$ is negative), LTP occurs in the gray region.

\section{Numerical integration}

Numerical integration of stochastic differential equations (2)-(4) is done using the Heun method (San Miguel and Toral 2000) (with the time step $\Delta t=0.01 \mathrm{~ms}$ ). For each realization of the stochastic process, we choose a random initial point $\left[x_{i}(0), y_{i}(0), z_{i}(0)\right]$ for the $i$ th $(i=1, \ldots, N)$ neuron with uniform probability in the range of $x_{i}(0) \in(-1.5,1.5), \quad y_{i}(0) \in(-10,0), \quad$ and $z_{i}(0) \in(1.2,1.5)$.

\section{Effects of inhibitory STDP on burst synchronization}

\section{BS in the absence of iSTDP}

First, we are concerned about the BS (i.e., population synchronization on the slow bursting timescale) in the absence of iSTDP for the case of symmetric attachment with $l_{\text {in }}=l_{\text {out }}=l^{*}=15$ in the SFN of $N$ inhibitory Hindmarsh-Rose bursting neurons. The coupling strengths $\left\{J_{i j}\right\}$ are static, and their values are chosen from the Gaussian distribution where the mean $J_{0}$ is 12 and the standard deviation $\sigma_{0}$ is 0.1 . We investigate emergence of BS by varying the noise intensity $D$.

BS may be well visualized in the raster plot of burst onset times which corresponds to a collection of all trains of burst onset times of individual bursting neurons. Figure 3a1-a5 show such raster plots for various values of $D$. To see emergence of BS, we employ an (experimentally-obtainable) instantaneous population burst rate (IPBR) which is often used as a collective quantity showing bursting behaviors. This IPBR may be obtained from the raster plot of burst onset times (Kim and Lim 2015a, b, 2016). To obtain a smooth IPBR, we employ the kernel density estimation (kernel smoother) (Shimazaki and Shinomoto 2010). Each burst onset time in the raster plot is convoluted (or blurred) with a kernel function $K_{h}(t)$ to obtain a smooth estimate of IPBR $R_{b}(t)$ :

$R_{b}(t)=\frac{1}{N} \sum_{i=1}^{N} \sum_{b=1}^{n_{i}} K_{h}\left(t-t_{b}^{(i)}\right)$

where $t_{b}^{(i)}$ is the $b$ th burst onset time of the $i$ th neuron, $n_{i}$ is the total number of burst onset times for the $i$ th neuron, and we use a Gaussian kernel function of band width $h$ : 

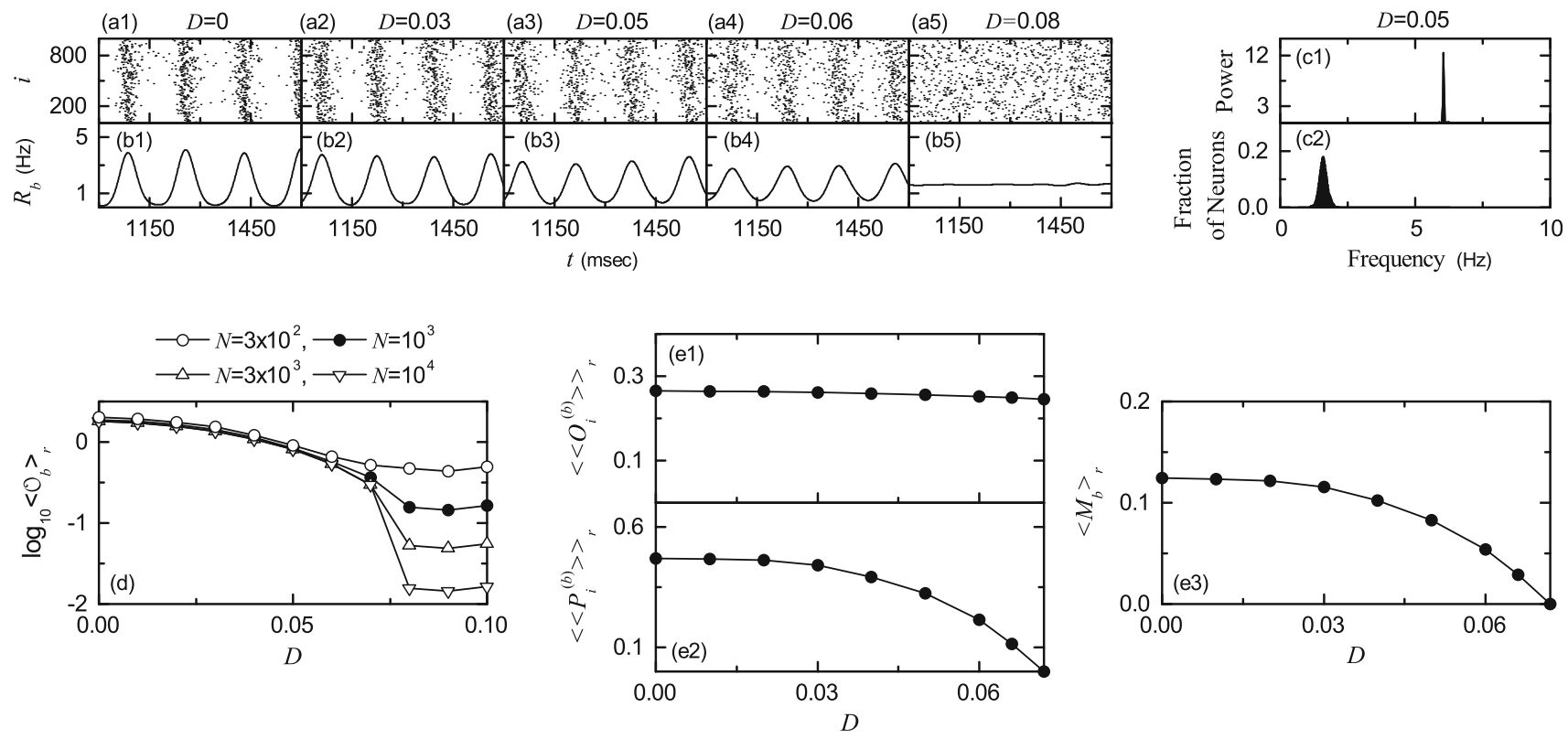

Fig. 3 Burst synchronization in the absence of iSTDP for the case of symmetric attachment with $l^{*}=15 ; N=10^{3}$ except for the case of the bursting order parameter in $\mathbf{d}$. Raster plots of burst onset times in a1-a5 and IPBR kernel estimates $R_{b}(t)$ in b1-b5 for various values of $D=0,0.03,0.05,0.06$, and 0.08 . c1 One-sided power spectrum of $\Delta R_{b}(t)\left[=R_{b}(t)-\overline{R_{b}(t)}\right]$ (the overbar represents the time average) with mean-squared amplitude normalization and $\mathbf{c 2}$ distribution of

$K_{h}(t)=\frac{1}{\sqrt{2 \pi} h} e^{-t^{2} / 2 h^{2}}, \quad-\infty<t<\infty$

Throughout the paper, the band width $h$ of $K_{h}(t)$ is $20 \mathrm{~ms}$. Figure 3b1-b5 show IPBR kernel estimates $R_{b}(t)$ for various values of $D$. For the synchronous case, "bursting stripes" (composed of burst onset times and indicating BS) are formed in the raster plot of burst onset times (see Fig. 3a1-a4), and the corresponding IPBR kernel estimates $R_{b}(t)$ exhibit oscillations, as shown in Fig. 3b1-b4. As an example, we consider the case of $D=0.05$. The bursting frequency $f_{b}$ [i.e., the oscillating frequency of $R_{b}(t)$ ] is $6.09 \mathrm{~Hz}$ (see Fig. 3c1), while the population-averaged mean bursting rate (MBR) $\left\langle f_{i}^{(b)}\right\rangle$ of individual bursting neurons is $1.56 \mathrm{~Hz}$ (see Fig. 3c2). For this type of BS, individual bursting neurons fire at lower rates $f_{i}^{(b)}$ than the bursting frequency $f_{b}$, and hence only a smaller fraction of bursting neurons fire in each bursting stripe in the raster plot (i.e., each stripe is sparsely occupied by burst onset times of a smaller fraction of bursting neurons). In this way, sparse BS occurs, in contrast the full BS where individual neurons show bursting at every global cycle of $R_{b}(t)$ (Kim and Lim 2018b). On the other hand, in the desynchronized case for $D>D^{*}(\simeq 0.072)$, burst onset times are completely scattered in the raster plot, and $R_{b}(t)$ is nearly stationary, as shown in Fig. 3a5, b5 for $D=0.08$. mean bursting rates (MBRs) of individual neurons for $D=0.05$. d Plots of the thermodynamic bursting order parameter $\left\langle\mathcal{O}_{b}\right\rangle_{r}$ versus $D$. Plots of $\mathbf{e 1}$ the average occupation degree $\left\langle\left\langle O_{i}^{(b)}\right\rangle\right\rangle_{r}$, e2 the average pacing degree $\left\langle\left\langle P_{i}^{(b)}\right\rangle\right\rangle_{r}$, and $\mathbf{e} \mathbf{3}$ the statistical-mechanical bursting measure $\left\langle M_{b}\right\rangle_{r}$ versus $D$

Recently, we introduced a realistic bursting order parameter, based on $R_{b}(t)$, for describing transition from BS to desynchronization (Kim and Lim 2015b). The mean square deviation of $R_{b}(t)$,

$\mathcal{O}_{b} \equiv \overline{\left(R_{b}(t)-\overline{R_{b}(t)}\right)^{2}}$,

plays the role of an order parameter $\mathcal{O}_{b}$; the overbar represents the time average. This bursting order parameter may be regarded as a thermodynamic measure because it concerns just the macroscopic IPBR kernel estimate $R_{b}(t)$ without any consideration between $R_{b}(t)$ and microscopic individual burst onset times. In the thermodynamic limit of $N \rightarrow \infty$, the bursting order parameter $\mathcal{O}_{b}$ approaches a non-zero (zero) limit value for the synchronized (desynchronized) state. Hence, the bursting order parameter can determine synchronized and desynchronized states. Figure $3 \mathrm{~d}$ shows plots of $\log _{10}\left\langle\mathcal{O}_{b}\right\rangle_{r}$ versus $D$. In each realization, we discard the first time steps of a stochastic trajectory as transients for $10^{3} \mathrm{~ms}$, and then we numerically compute $\mathcal{O}_{b}$ by following the stochastic trajectory for $3 \times 10^{4} \mathrm{~ms}$. Hereafter, $\langle\cdots\rangle_{r}$ denotes an average over 20 realizations. For $D<D^{*}(\simeq 0.072)$, the bursting order parameter $\left\langle\mathcal{O}_{b}\right\rangle_{r}$ approaches a non-zero limit value, and hence BS appears. On the hand hand, when passing $D^{*}$ a transition from BS to desynchronization occurs, because $\left\langle\mathcal{O}_{b}\right\rangle_{r}$ tends to zero with increasing $N$. 
In the absence of noise (i.e., $D=0$ ), sparse bursting stripes (indicating sparse BS) appear successively in the raster plot of burst onset times, and the IPBR kernel estimate $R_{b}(t)$ exhibits an oscillatory behavior. However, as $D$ is increased, sparse bursting stripes become smeared gradually, as shown in the cases of $D=0.03,0.05$, and 0.06 , and hence the amplitudes of $R_{b}(t)$ decrease in a slow way. Eventually, as $D$ passes $D^{*}$, desynchronization occurs due to overlap of smeared bursting stripes. Then, burst onset times are completely scattered without forming any bursting stripes, and hence the IPBR kernel estimate $R_{b}(t)$ becomes nearly stationary, as shown for the case of $D=0.08$.

We characterize sparse BS in the range of $0 \leq D<D^{*}$ by employing a statistical-mechanical bursting measure $M_{b}$ (Kim and Lim 2015b). For the case of BS, bursting stripes appear regularly in the raster plot of burst onset times. The bursting measure $M_{i}^{(b)}$ of the $i$ th bursting stripe is defined by the product of the occupation degree $O_{i}^{(b)}$ of burst onset times (denoting the density of the $i$ th bursting stripe) and the pacing degree $P_{i}^{(b)}$ of burst onset times (representing the smearing of the $i$ th bursting stripe):

$M_{i}^{(b)}=O_{i}^{(b)} \cdot P_{i}^{(b)}$.

The occupation degree $O_{i}^{(b)}$ of burst onset times in the $i$ th bursting stripe is given by the fraction of bursting neurons:

$O_{i}^{(b)}=\frac{N_{i}^{(b)}}{N}$

where $N_{i}^{(b)}$ is the number of bursting neurons in the $i$ th bursting stripe. For the case of full BS, all bursting neurons exhibit burstings in each bursting stripe in the raster plot of burst onset times, and hence the occupation degree $O_{i}^{(b)}$ of Eq. (13) in each bursting stripe becomes 1. On the other hand, in the case of sparse BS, only some fraction of bursting neurons show burstings in each bursting stripe, and hence the occupation degree $O_{i}^{(b)}$ becomes less than 1 . In our case of BS, $O_{i}^{(b)}<1$ in the range of $0 \leq D<D^{*}$, and hence sparse BS occurs. The pacing degree $P_{i}^{(b)}$ of burst onset times in the $i$ th bursting stripe can be determined in a statistical-mechanical way by taking into account their contributions to the macroscopic IPBR kernel estimate $R_{b}(t)$. Central maxima of $R_{b}(t)$ between neighboring left and right minima of $R_{b}(t)$ coincide with centers of bursting stripes in the raster plot. A global cycle starts from a left minimum of $R_{b}(t)$, passes a maximum, and ends at a right minimum. An instantaneous global phase $\Phi^{(b)}(t)$ of $R_{b}(t)$ was introduced via linear interpolation in the region forming a global cycle [for details, refer to Eqs. (14) and (15) in Kim and Lim (2015b)]. Then, the contribution of the $k$ th microscopic burst onset time in the $i$ th bursting stripe occurring at the time $t_{k}^{(b)}$ to $R_{b}(t)$ is given by $\cos \Phi_{k}^{(b)}$, where $\Phi_{k}^{(b)}$ is the global phase at the $k$ th burst onset time [i.e., $\Phi_{k}^{(b)} \equiv \Phi^{(b)}\left(t_{k}^{(b)}\right)$ ]. A microscopic burst onset time makes the most constructive (in-phase) contribution to $R_{b}(t)$ when the corresponding global phase $\Phi_{k}^{(b)}$ is $2 \pi n$ ( $n=0,1,2, \ldots)$, while it makes the most destructive (antiphase) contribution to $R_{b}(t)$ when $\Phi_{k}^{(b)}$ is $2 \pi(n-1 / 2)$. By averaging the contributions of all microscopic burst onset times in the $i$ th bursting stripe to $R_{b}(t)$, we obtain the pacing degree of burst onset times in the $i$ th stripe:

$P_{i}^{(b)}=\frac{1}{B_{i}} \sum_{k=1}^{B_{i}} \cos \Phi_{k}^{(b)}$,

where $B_{i}$ is the total number of microscopic burst onset times in the $i$ th stripe.

By averaging $M_{i}^{(b)}$ of Eq. (12) over a sufficiently large number $N_{b}$ of bursting stripes, we obtain the realistic statistical-mechanical bursting measure $M_{b}$, based on the IPBR kernel estimate $R_{b}(t)$ :

$M_{b}=\frac{1}{N_{b}} \sum_{i=1}^{N_{b}} M_{i}^{(b)}$.

We follow $3 \times 10^{3}$ bursting stripes in each realization and get $\left\langle M_{b}\right\rangle_{r}$ via average over 20 realizations. Figure 3e1-e3 show the average occupation degree $\left\langle\left\langle O_{i}^{(b)}\right\rangle\right\rangle_{r}$, the average pacing degree $\left\langle\left\langle P_{i}^{(b)}\right\rangle\right\rangle_{r}$, and the statistical-mechanical bursting measure $\left\langle M_{b}\right\rangle_{r}$, respectively. With increasing $D$ from 0 to $D^{*},\left\langle\left\langle O_{i}^{(b)}\right\rangle\right\rangle_{r}$ (denoting the density of bursting stripes in the raster plot) decreases very slowly from 0.27 to 0.25 (i.e., $\left\langle\left\langle O_{i}^{(b)}\right\rangle\right\rangle_{r}$ is nearly constant). The average pacing degree $\left\langle\left\langle P_{i}^{(b)}\right\rangle\right\rangle_{r}$ represents well the smearing degree of bursting stripes in the raster plot of burst onset times (shown in Fig. 3a1-a4). With increasing $D$ from 0 to $D^{*}$, $\left\langle\left\langle P_{i}^{(b)}\right\rangle\right\rangle_{r}$ decreases to zero smoothly due to complete overlap of sparse bursting stripes. Through product of the average occupation and pacing degrees of burst onset times, the statistical-mechanical bursting measure $\left\langle M_{b}\right\rangle_{r}$ is obtained. Since $\left\langle\left\langle O_{i}^{(b)}\right\rangle\right\rangle_{r}$ is nearly constant, $\left\langle M_{b}\right\rangle_{r}$ behaves like the case of $\left\langle\left\langle P_{i}^{(b)}\right\rangle\right\rangle_{r}$.

We now fix the value of $D$ at $D=0.05$ where BS occurs for the case of symmetric attachment with $l^{*}=15$ (see Fig. 3a3, b3), and investigate the effect of scale-free connectivity on BS by varying the degree of symmetric attachment $l^{*}$ (i.e., $l_{\text {in }}=l_{\text {out }}=l^{*}$ ) and the asymmetry parameter $\Delta l$ of asymmetric attachment [i.e., $l_{\text {in }}=l^{*}+\Delta l$ and $\left.l_{\text {out }}=l^{*}-\Delta l\left(l^{*}=15\right)\right]$. 
We first consider the case of symmetric attachment, and study its effect on BS by varying the degree $l^{*}$. Figure 4a1a5 show the raster plots of burst onset times for various values of $l^{*}$. Their corresponding IPBR kernel estimates $R_{b}(t)$ are also shown in Fig. 4b1-b5. With increasing $l^{*}$ from 15 (i.e., the case studied above), bursting stripes in the raster plots of burst onset times become clearer (e.g., see the cases of $l^{*}=23$ and 30), which also leads to increase in the oscillating amplitudes of $R_{b}(t)$, in comparison with that for the case of $l^{*}=15$. In this way, as $l^{*}$ is increased from
15, the degree of BS becomes better. On the other hand, with decreasing $l^{*}$ from 15 , bursting stripes become more smeared (e.g., see the case of $l^{*}=12$ ), and hence the oscillating amplitude of $R_{b}(t)$ decreases. Thus, as $l^{*}$ is decreased from 15, the degree of BS becomes worse. Eventually, the population state becomes desynchronized for $l^{*} \leq 7$, as shown in Fig. 4a1, b1 where burst onset times are completely scattered and $R_{b}(t)$ becomes nearly stationary.
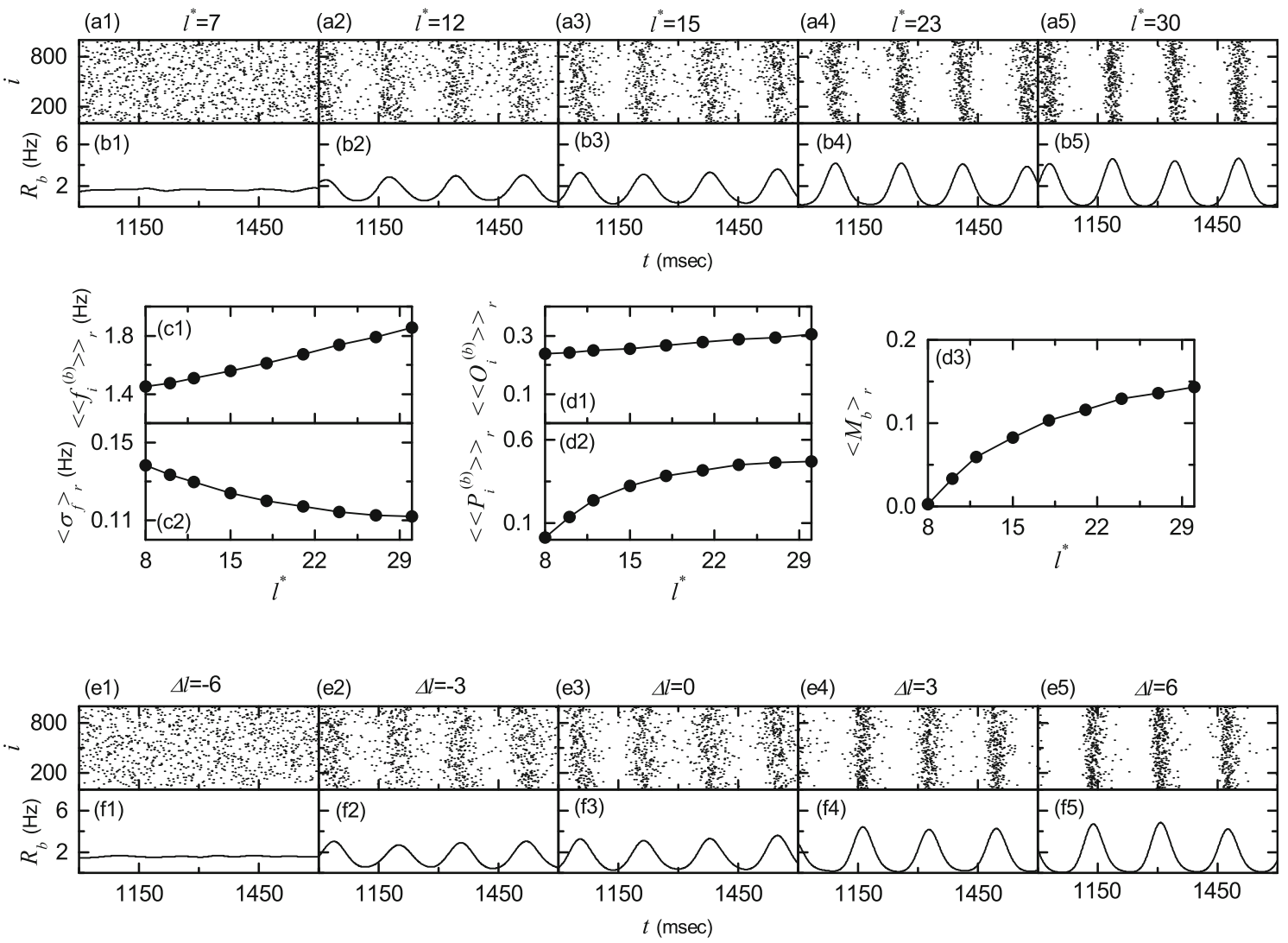

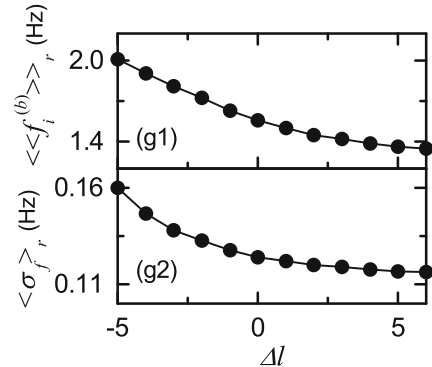

Fig. 4 Effect of network architecture on BS in the absence of iSTDP for $D=0.05 ; N=10^{3}$. Symmetric preferential attachment with $l_{\text {in }}=l_{\text {out }}=l^{*}$. Raster plots of burst onset times in a1-a5 and IPBR kernel estimates $R_{b}(t)$ in b1-b5 for various values of $l^{*}$. Plots of $\mathbf{c 1}$ population-averaged MBRs $\left\langle\left\langle f_{i}^{(b)}\right\rangle\right\rangle_{r}$ and $\mathbf{c 2}$ standard deviations $\left\langle\sigma_{f}\right\rangle_{r}$ from $\left\langle f_{i}^{(b)}\right\rangle$ versus $l^{*}$. Plots of $\mathbf{d 1}$ the average occupation degree $\left\langle\left\langle O_{i}^{(b)}\right\rangle\right\rangle_{r}$, d2 the average pacing degree $\left\langle\left\langle P_{i}^{(b)}\right\rangle\right\rangle_{r}$, and $\mathbf{d 3}$ the statistical-mechanical bursting measure $\left\langle M_{b}\right\rangle_{r}$ versus $l^{*}$. Asymmetric

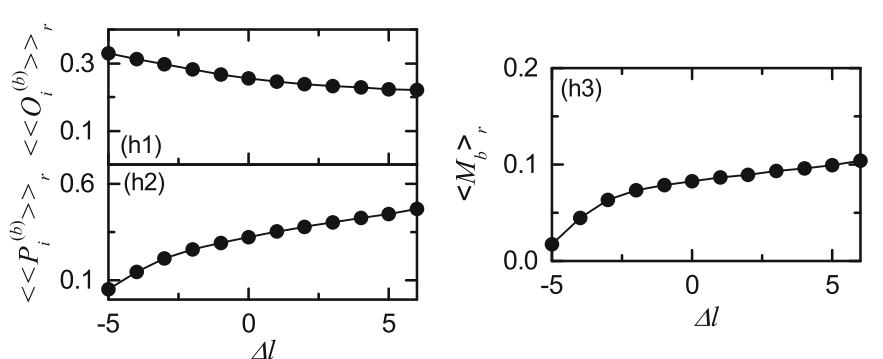

preferential attachment with $l_{\text {in }}=l^{*}+\Delta l$ and $l_{\text {out }}=l^{*}-\Delta l\left(l^{*}=15\right)$. Raster plots of burst onset times in e1-e5 and IPBR kernel estimates $R_{b}(t)$ in f1-f5 for various values of $\Delta l$. Plots of g1 populationaveraged MBRs $\left\langle\left\langle f_{i}^{(b)}\right\rangle\right\rangle_{r}$ and $\mathbf{g} 2$ standard deviations $\left\langle\sigma_{f}\right\rangle_{r}$ from $\left\langle f_{i}^{(b)}\right\rangle$ versus $\Delta l$. Plots of $\mathbf{h} \mathbf{1}$ the average occupation degree $\left\langle\left\langle O_{i}^{(b)}\right\rangle\right\rangle_{r}, \mathbf{h} \mathbf{2}$ the average pacing degree $\left\langle\left\langle P_{i}^{(b)}\right\rangle\right\rangle_{r}$, and $\mathbf{h} \mathbf{3}$ the statistical-mechanical bursting measure $\left\langle M_{b}\right\rangle_{r}$ versus $\Delta l$ 
Effects of $l^{*}$ on network topology were characterized in Kim and Lim (2016), where the group properties of the SFN were studied in terms of the average path length $L_{p}$ and the betweenness centralization $B_{c}$ by varying $l^{*}$. The average path length $L_{p}$ represents typical separation between two nodes in the network, and it characterizes global efficiency of information transfer between distant nodes (Albert and Barabási 2002; Kim and Lim 2016). The group betweenness centralization $B_{c}$ denotes how much the load of communication traffic is concentrated on the head hub (with the highest degree) (Freeman 1977, 1978; Kim and Lim 2016). Large $B_{c}$ implies that load of communication traffic is much concentrated on the head hub, and hence the head hub tends to become overloaded by the communication traffic passing through it (Nishikawa et al. 2003). With increasing $l^{*}$, both the average path length $L_{p}$ and the betweenness centralization $B_{c}$ become smaller, due to increase in the total number of connections [see Fig. 11c, e in Kim and Lim (2016)]. Hence, typical separation between neurons becomes shorter, and load of communication traffic concentrated on the head neuron also becomes smaller. As a result, as $l^{*}$ is increased, efficiency of global communication between neurons (i.e., global transfer of neural information between neurons via synaptic connections) becomes better, which may contribute to increase in the degree of BS.

Along with network topology, we also consider individual dynamics which change depending on the synaptic inputs with the in-degree $d^{(i n)}$ of Eq. (6). The in-degree distribution affects MBRs of individual bursting neurons [e.g., see Fig. 11g1-g5 in Kim and Lim (2016)]. As $l^{*}$ is increased, the average in-degree $\left\langle d^{(i n)}\right\rangle\left(=\frac{1}{N} \sum_{i=1}^{N} d_{i}^{(i n)}\right)$ increases, which favors the pacing between bursting neurons. Consequently, with increasing $l^{*}$, the population-averaged MBR $\left\langle\left\langle f_{i}^{(b)}\right\rangle\right\rangle_{r}$ increases, and the standard deviation $\left\langle\sigma_{f}\right\rangle_{r}$ decreases (i.e., population-averaged individual dynamics become better), as shown in Fig. 4c1, c2, which may also contribute to increase in the degree of BS. Figure $4 \mathrm{~d} 1-\mathrm{d} 3$ show the average occupation degree $\left\langle\left\langle O_{i}^{(b)}\right\rangle\right\rangle_{r}$, the average pacing degree $\left\langle\left\langle P_{i}^{(b)}\right\rangle\right\rangle_{r}$, and the statisticalmechanical bursting measure $\left\langle M_{b}\right\rangle_{r}$, respectively. With increasing $l^{*},\left\langle\left\langle P_{i}^{(b)}\right\rangle\right\rangle_{r}$ increases markedly due to decrease in the standard deviation $\left\langle\sigma_{f}\right\rangle_{r}$. On the other hand, $\left\langle\left\langle O_{i}^{(b)}\right\rangle\right\rangle_{r}$ increases a little due to a slight increase in the populationaveraged MBR $\left\langle\left\langle f_{i}^{(b)}\right\rangle\right\rangle_{r}$. Then, $\left\langle M_{b}\right\rangle_{r}$ (given by the product of $\left\langle\left\langle O_{i}^{(b)}\right\rangle\right\rangle_{r}$ and $\left\langle\left\langle P_{i}^{(b)}\right\rangle\right\rangle_{r}$ ) increases distinctly as in the case of $\left\langle\left\langle P_{i}^{(b)}\right\rangle\right\rangle_{r}$. Consequently, as $l^{*}$ is increased from 15 , the degree of $\mathrm{BS}$ increases due to both better individual dynamics and better efficiency of global communication between nodes (resulting from the increased number of total connections). On the other hand, with decreasing $l^{*}$ from 15, both individual dynamics and effectiveness of communication between nodes become worse (resulting from the decreased number of total connections), and hence the degree of BS decreases.

As the second case of network architecture, we consider the case of asymmetric attachment; $l_{i n}=l^{*}+\Delta l$ and $l_{\text {out }}=l^{*}-\Delta l\left(l^{*}=15\right)$. For the case of asymmetric attachment, the total number of inward and outward connections is fixed (i.e., $l_{\text {in }}+l_{\text {out }}=30=$ constant), in contrast to the case of symmetric attachment where with increasing $l^{*}$ the number of total connections increases. We investigate the effect of asymmetric attachment on BS by varying the asymmetry parameter $\Delta l$.

Figure 4e1-e5 show the raster plots of burst onset times for various values of $\Delta l$. Their corresponding IPBR kernel estimates $R_{b}(t)$ are also shown in Fig. 4f1-f5. As $\Delta l$ is increased from 0 , bursting stripes in the raster plots of burst onset times become clearer (e.g., see the cases of $\Delta l=3$ and 6), and hence the oscillating amplitudes of $R_{b}(t)$ become larger than that for the case of $\Delta l=0$. In this way, with increasing $\Delta l$ from 0 , the degree of $\mathrm{BS}$ becomes better. On the other hand, with decreasing $\Delta l$ from 0 , bursting stripes become more smeared (e.g., see the case of $\Delta l=-3)$, which results in decrease in the oscillating amplitudes of $R_{b}(t)$. Hence, as $\Delta l$ is decreased from 0 , the degree of BS becomes worse. Eventually, the population state becomes desynchronized for $\Delta l \leq-6$, as shown in Fig. $4 \mathrm{e} 1$, f1 where burst onset times are completely scattered and $R_{b}(t)$ becomes nearly stationary.

As $|\Delta l|$ (the magnitude of $\Delta l$ ) is increased, both $L_{p}$ and $B_{c}$ increase symmetrically, independently of the sign of $\Delta l$, due to increased mismatching between the in- and the outdegrees [see Fig. 13c, d in Kim and Lim (2016)]. The values of $L_{p}$ and $B_{c}$ for both cases of different signs but the same magnitude (i.e., $\Delta l$ and $-\Delta l$ ) become the same because both inward and outward connections are involved equally in computations of $L_{p}$ and $B_{c}$. Due to the effects of $\Delta l$ on $L_{p}$ and $B_{c}$, with increasing $|\Delta l|$, efficiency of global communication between nodes becomes worse, independently of the sign of $\Delta l$, which may contribute to decrease in the degree of BS.

However, individual dynamics change depending on the sign of $\Delta l$ because of different average in-degrees $\left\langle d^{(i n)}\right\rangle$. Particularly, the distribution of MBRs of individual bursting neurons vary depending on $\Delta l$ [e.g., see Fig. $13 \mathrm{~g} 1-\mathrm{g} 5$ in $\mathrm{Kim}$ and $\mathrm{Lim}$ (2016)]. As $\Delta l$ is increased from $0,\left\langle d^{(i n)}\right\rangle$ increases, which tends to favor the pacing between bursting neurons. Hence, the standard deviation $\left\langle\sigma_{f}\right\rangle_{r}$ (for the distribution of MBRs $\left\{f_{i}^{(b)}\right\}$ ) decreases, as shown in Fig. 4g2. In addition, with increasing $\Delta l$ from 0 , the population-averaged MBR $\left\langle\left\langle f_{i}^{(b)}\right\rangle\right\rangle_{r}$ also decreases, because of increase in 
average inhibition given to individual neurons (resulting from increased population-averaged in-degrees) (see Fig. $4 \mathrm{~g} 1$ ). In contrast, with decreasing $\Delta l$ from 0 , the average in-degree $\left\langle d^{(i n)}\right\rangle$ decreases, which tends to disfavor the pacing between bursting neurons. Therefore, the standard deviation $\left\langle\sigma_{f}\right\rangle_{r}$ increases (see Fig. 4g2). Moreover, as $\Delta l$ is decreased from 0 , the population-averaged MBR $\left\langle\left\langle f_{i}^{(b)}\right\rangle\right\rangle_{r}$ also increases, due to decrease in average inhibition given to individual neurons (resulting from decreased population-averaged in-degrees) (see Fig. $4 \mathrm{~g} 1$ ). In this way, as $\Delta l$ is increased (decreased) from 0 , individual dynamics become better (worse), which may contribute to increase (decrease) in the degree of BS. Figure 4h1-h3 show the average occupation degree $\left\langle\left\langle O_{i}^{(b)}\right\rangle\right\rangle_{r}$, the average pacing degree $\left\langle\left\langle P_{i}^{(b)}\right\rangle\right\rangle_{r}$, and the statistical-mechanical bursting measure $\left\langle M_{b}\right\rangle_{r}$, respectively. With increasing $\Delta l$, $\left\langle\left\langle P_{i}^{(b)}\right\rangle\right\rangle_{r}$ increases distinctly, mainly due to decrease in the standard deviation $\left\langle\sigma_{f}\right\rangle_{r}$ (which overcomes worse efficiency of communication). In contrast, as $\Delta l$ is increased, $\left\langle\left\langle O_{i}^{(b)}\right\rangle\right\rangle_{r}$ decreases a little due to a slight decrease in the population-averaged MBR $\left\langle\left\langle f_{i}^{(b)}\right\rangle\right\rangle_{r}$. However, $\left\langle\left\langle P_{i}^{(b)}\right\rangle\right\rangle_{r}$ increases more rapidly than decrease in $\left\langle\left\langle O_{i}^{(b)}\right\rangle\right\rangle_{r}$. Hence, with increasing $\Delta l\left\langle M_{b}\right\rangle_{r}$ (given by the product of $\left\langle\left\langle O_{i}^{(b)}\right\rangle\right\rangle_{r}$ and $\left\langle\left\langle P_{i}^{(b)}\right\rangle\right\rangle_{r}$ ) also increases. Consequently, as $\Delta l$ is decreased from 0 , the degree of BS decreases because both individual dynamics and efficiency of communication between nodes are worse. On the other hand, with increasing $\Delta l$ from 0 , the degree of BS increases mainly because of better individual dynamics overcoming worse efficiency of communication.

\section{Effects of iSTDP on BS}

In this subsection, we study the effect of iSTDP on BS [occurring for $0 \leq D<D^{*}(\simeq 0.072)$ in the absence of iSTDP]. The initial values of synaptic strengths $\left\{J_{i j}\right\}$ are chosen from the Gaussian distribution with the mean $J_{0}$ (= 12 ) and the standard deviation $\sigma_{0}(=0.1)$. Here, we employ an anti-Hebbian time window for the synaptic modification $\Delta J_{i j}\left(\Delta t_{i j}\right)$ of Eq. (8). Then, $J_{i j}$ for each synapse is updated according to a nearest-burst pair-based STDP rule of Eq. (7).

We first consider the case of symmetric attachment with $l^{*}=15$, and investigate the effect of iSTDP on BS by varying $D$. Figure 5a shows time-evolutions of populationaveraged synaptic strengths $\left\langle J_{i j}\right\rangle$ for various values of $D$; $\langle\cdots\rangle$ represents an average over all synapses. For each case of $D=0,0.03$, and $0.05,\left\langle J_{i j}\right\rangle$ decreases monotonically below its initial value $J_{0}(=12)$, and it approaches a saturated limit value $\left\langle J_{i j}^{*}\right\rangle$ nearly at $t=1000 \mathrm{~s}$. Consequently,
LTD occurs for these values of $D$. On the other hand, for $D=0.06\left\langle J_{i j}\right\rangle$ increases monotonically above $J_{0}$, and approaches a saturated limit value $\left\langle J_{i j}^{*}\right\rangle$. As a result, LTP occurs for the case of $D=0.06$. Histograms for fraction of synapses versus $J_{i j}^{*}$ (saturated limit values of $J_{i j}$ at $t=1000 \mathrm{~s}$ ) are shown in black color for various values of $D$ in Fig. 5b1-b4; the bin size for each histogram is 0.1 . For comparison, initial distributions of synaptic strengths $\left\{J_{i j}\right\}$ (i.e., Gaussian distributions whose mean $J_{0}$ and standard deviation $\sigma_{0}$ are 12 and 0.1 , respectively) are also shown in gray color. For the cases of LTD $(D=0,0.03$, and 0.05), their black histograms lie on the left side of the initial gray histograms, and hence their population-averaged values $\left\langle J_{i j}^{*}\right\rangle$ become smaller than the initial value $J_{0}$. On the other hand, the black histogram for the case of LTP $(D=0.06)$ is shifted to the right side of the initial gray histogram, and hence its population-averaged value $\left\langle J_{i j}^{*}\right\rangle$ becomes larger than $J_{0}$. For both cases of LTD and LTP, their black histograms are wider than the initial gray histograms [i.e., the standard deviations $\sigma_{J}$ are larger than the initial one $\left.\sigma_{0}\right]$. Figure $5 \mathrm{c} 1$ shows a plot of population-averaged limit values of synaptic strengths $\left\langle\left\langle J_{i j}^{*}\right\rangle\right\rangle_{r}$ versus $D$. Here, the horizontal dotted line represents the initial average value of coupling strengths $J_{0}$, and the threshold value $D_{t h}(\simeq 0.0558)$ for LTD/LTP (where $\left.\left\langle\left\langle J_{i j}^{*}\right\rangle\right\rangle_{r}=J_{0}\right)$ is represented by a solid circle. Hence, LTD occurs in a range of BS $\left(0 \leq D<D_{t h}\right)$; BS in the absence of iSTDP appears in the range of $0 \leq D<D^{*}$. As $D$ is decreased from $D_{t h}$, $\left\langle\left\langle J_{i j}^{*}\right\rangle\right\rangle_{r}$ decreases monotonically. In contrast, LTP takes place in a smaller range of BS (i.e., $D_{t h}<D<D^{*}$ ), and with increasing $D$ from $D_{t h}\left\langle\left\langle J_{i j}^{*}\right\rangle\right\rangle_{r}$ increases monotonically. Figure $5 \mathrm{c} 2$ shows plots of standard deviations $\left\langle\sigma_{J}\right\rangle_{r}$ versus $D$. With increasing $D$ from 0 to $D^{*},\left\langle\sigma_{J}\right\rangle_{r}$ increases, and all the values of $\left\langle\sigma_{J}\right\rangle_{r}$ are larger than the initial value $\sigma_{0}(=0.1)$.

The LTD (LTP) has a tendency to increase (decrease) the degree of BS because of decrease (increase) in the mean value of synaptic inhibition strengths, and the increased standard deviations tend to decrease the degree of BS. The effects of LTD and LTP on BS after the saturation time $(t=1000 \mathrm{~s})$ may be well shown in the raster plot of burst onset times and the corresponding IPBR kernel estimate $R_{b}(t)$, which are given in Fig. 6a1-a4, b1b4 for various values of $D$, respectively. When compared with Fig. 3a1-a3, b1-b3 in the absence of iSTDP, the degrees of BS for the case of $D=0,0.03$, and 0.05 are increased [i.e., the amplitudes of $R_{b}(t)$ are increased] due to dominant effect of LTD (overcoming the effect of increased standard deviation). On the other hand, in the case of $D=0.06$ the population state becomes desynchronized [i.e., $R_{b}(t)$ becomes nearly stationary] due to the 

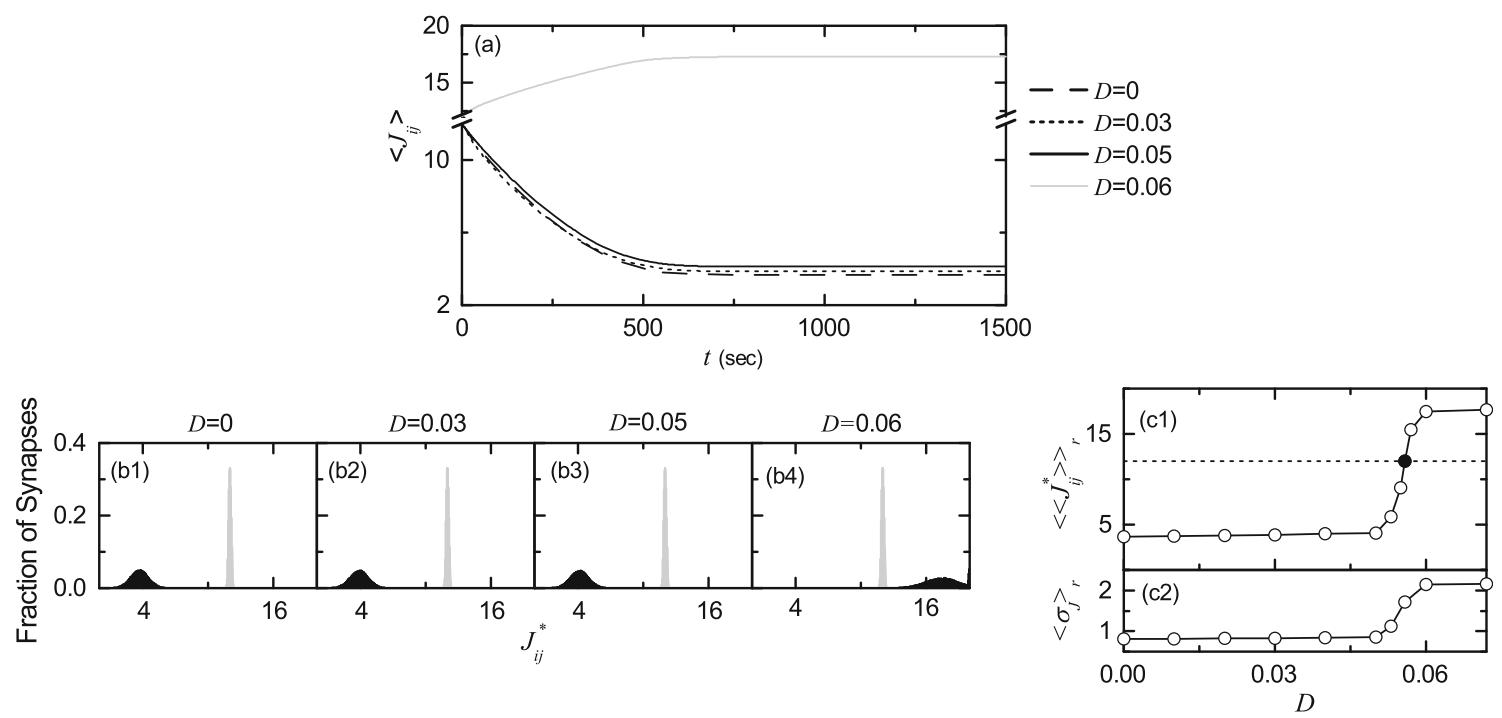

Fig. 5 Effect of iSTDP on BS for the case of symmetric attachment with $l^{*}=15 ; N=10^{3}$. a Time-evolutions of population-averaged synaptic strengths $\left\langle J_{i j}\right\rangle$ for various values of $D$. b1-b4 Histograms for the fraction of synapses versus $J_{i j}^{*}$ (saturated limit values of $J_{i j}$ at
$1000 \mathrm{~s}$ ) are shown in black color for various values of $D$; for comparison, initial distributions of synaptic strengths $\left\{J_{i j}\right\}$ are also shown in gray color. Plots of $\mathbf{c 1}$ population-averaged limit values of synaptic strengths $\left\langle\left\langle J_{i j}^{*}\right\rangle\right\rangle_{r}$ and c2 standard deviations $\left\langle\sigma_{J}\right\rangle_{r}$ versus $D$
Fig. 6 Characterization of BS after the saturation time $(t=1000 \mathrm{~s})$ for the case of symmetric attachment with $l^{*}=15 ; N=10^{3}$. Raster plots of burst onset times in $\mathbf{a 1}-\mathbf{a} 4$ and IPBR kernel estimates $R_{b}(t)$ in b1-b4 for various values of $D$ after the saturation time, where $t=t^{*}$ (saturation time $=1000$ s) $+\widetilde{t}$. Plots of $\mathbf{c 1}$ the average occupation degree $\left\langle\left\langle O_{i}^{(b)}\right\rangle\right\rangle_{r}$ (open circles), $\mathbf{c 2}$ the average pacing degree $\left\langle\left\langle P_{i}^{(b)}\right\rangle\right\rangle_{r}$ (open circles), and $\mathbf{c 3}$ the statisticalmechanical bursting measure $\left\langle M_{b}\right\rangle_{r}$ (open circles) versus $D$. For comparison, $\left\langle\left\langle O_{i}^{(b)}\right\rangle\right\rangle_{r}$, $\left\langle\left\langle P_{i}^{(b)}\right\rangle\right\rangle_{r}$, and $\left\langle M_{b}\right\rangle_{r}$ versus $D$ in the absence of iSTDP are also denoted by crosses
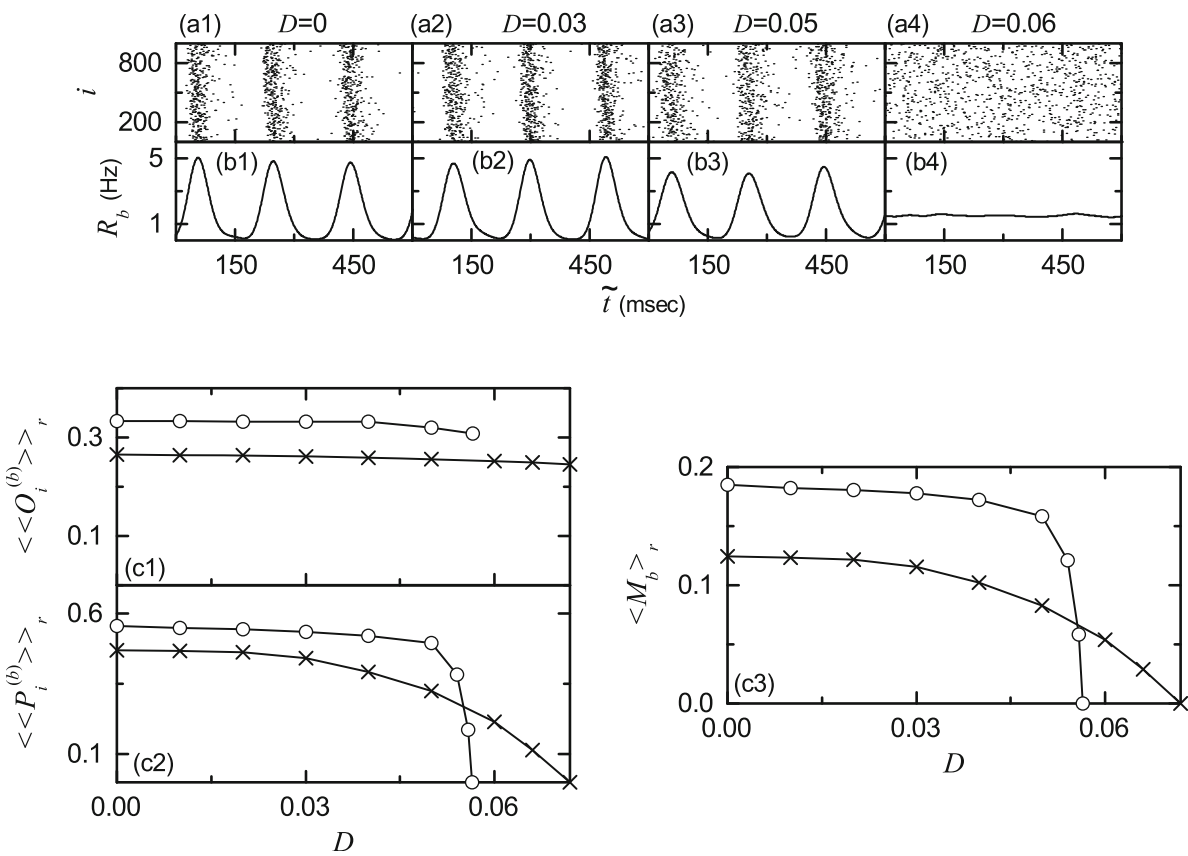

effects of both LTP and increased standard deviation (compare Fig. 6a4, b4 with Fig. 3a4, b4). Due to inhibition, the roles of LTD and LTP in inhibitory synaptic plasticity are reversed in comparison with those in excitatory synaptic plasticity where the degree of population synchronization is increased (decreased) via LTP (LTD) (Kim and $\operatorname{Lim} 2018 \mathrm{a}, \mathrm{b}, \mathrm{c})$.

In the presence of iSTDP, we also characterize population behaviors for BS after the saturation time $(t=1000 \mathrm{~s})$ in the range of $0 \leq D<D^{* *}(\simeq 0.0565)$ (where
BS persists in the presence of iSTDP). For comparison, corresponding quantities for BS in the absence of iSTDP are also shown in the range of $0 \leq D<D^{*}(\simeq 0.072)$ (where BS appears in the absence of iSTDP). Figure $6 \mathrm{c} 1$, c 2 show the average occupation degree $\left\langle\left\langle O_{i}^{(b)}\right\rangle\right\rangle_{r}$ and the average pacing degree $\left\langle\left\langle P_{i}^{(b)}\right\rangle\right\rangle_{r}$ (represented by open circles), respectively; for comparison, $\left\langle\left\langle O_{i}^{(b)}\right\rangle\right\rangle_{r}$ and $\left\langle\left\langle P_{i}^{(b)}\right\rangle\right\rangle_{r}$ (denoted by crosses) are also shown in the case without iSTDP. In the region of $0 \leq D<D_{t h}(\simeq 0.0558)$, the values 
of $\left\langle\left\langle O_{i}^{(b)}\right\rangle\right\rangle_{r}$ (open circles) are larger than those (crosses) in the absence of iSTDP, due to LTD (decreased mean synaptic inhibition). In most region of LTD, the values of $\left\langle\left\langle P_{i}^{(b)}\right\rangle\right\rangle_{r}$ (open circles) are also larger than those (crosses) in the absence of iSTDP, because of dominant effect of LTD (overcoming the effect of increased standard deviation). In the region of $D_{t h}<D<D^{* *},\left\langle\left\langle O_{i}^{(b)}\right\rangle\right\rangle_{r}$ (open circles) decreases just a little. However, for the case of $\left\langle\left\langle P_{i}^{(b)}\right\rangle\right\rangle_{r}$ (open circles), a rapid transition to the case of $\left\langle\left\langle P_{i}^{(b)}\right\rangle\right\rangle_{r}=0$ occurs due to the effects of both LTP and increased standard deviation, in contrast to the smooth decrease in $\left\langle\left\langle P_{i}^{(b)}\right\rangle\right\rangle_{r}$ (crosses) in the absence of iSTDP. The statistical-mechanical bursting measure $\left\langle M_{b}\right\rangle_{r}$ (combining the effect of both the average occupation and pacing degrees) is shown in open circles in Fig. 6c3. Behaviors of $\left\langle M_{b}\right\rangle_{r}$ are similar to those of $\left\langle\left\langle P_{i}^{(b)}\right\rangle\right\rangle_{r}$, because the values of $\left\langle\left\langle O_{i}^{(b)}\right\rangle\right\rangle_{r}$ are nearly constant. A Matthew effect is found to occur in the presence of iSTDP. In most range of $D$ with LTD, good BS (with higher bursting measure) gets better since the effect of mean LTD is dominant in comparison to the effect of increased standard deviation. On the other hand, in the range of $D$ with LTP, bad BS (with lower bursting measure) gets worse because of the effects of both LTP and increased standard deviation. Thus, near the threshold $D_{t h}$ a rapid transition to desynchronization (i.e. the case of $\left\langle M_{b}\right\rangle_{r}=0$ ) occurs via LTP, in contrast to a smooth transition in the absence of iSTDP.

From now on, we make an intensive investigation on emergences of LTD and LTP of synaptic strengths via a microscopic method based on the distributions of time delays $\left\{\Delta t_{i j}\right\}$ between the pre- and the post-synaptic burst onset times. For understanding time-evolution of the distribution of $\left\{\Delta t_{i j}\right\}$, we first study the time-evolutions of the IBI histograms for $D=0.05$ (LTD) and 0.06 (LTP), which are shown in Fig. 7a1-a5, b1-b5, respectively. Here, we consider 5 stages, represented by I (starting from $0 \mathrm{~s}$ ), II (starting from $100 \mathrm{~s}$ ), III (starting from $250 \mathrm{~s}$ ), IV (starting from $500 \mathrm{~s}$ ), and V (starting from $800 \mathrm{~s}$ ). For each stage, we get the IBI histogram from IBIs obtained from all bursting neurons during $3 \mathrm{~s}$, and the bin size is $2.5 \mathrm{~ms}$. For the case of $D=0.05$ (LTD), the IBI histogram at the stage I consists of two peaks; the 1st lower peak and the 2nd higher peak, located at $3 T_{G}$ and $4 T_{G}\left[T_{G}\right.$ (denoted by the vertical dotted lines): global period of the IPBR $R_{b}(t)$ ], respectively. Hence, individual neurons exhibit burstings intermittently at every $3 \mathrm{rd}$ or 4 th global cycle of $R_{b}(t)$. Since the amplitude of the 2nd peak is larger, occurrence of burstings at every 4th global cycle is more probable. However, as the time $t$ is increased (i.e., with increase in the level of stage) the amplitude of the 1st peak increases, while that of the 2nd peak is reduced. In the stages IV and $\mathrm{V}$, extremely small 2nd peaks are shown in the insets. Hence, eventually individual neurons show burstings nearly at every 3 rd global cycle of $R_{b}(t)$. In the case of $D=0.06$ (LTP), the IBI histogram at the stage I is also composed of the 1st lower peak and the 2 nd higher peak, located at $3 T_{G}$ and $4 T_{G}$, respectively. However, with increasing the stage, peaks become wider, and merging between them occurs. Eventually, at the stage $\mathrm{V}$ a single broad peak appears. These distributions of IBIs for $D=$ 0.05 and 0.06 affect the distribution of $\left\{\Delta t_{i j}\right\}$, as shown below.

Figure 7c1-c5, d1-d5 show time-evolutions of normalized histograms $H\left(\Delta t_{i j}\right)$ for the distributions of time delays $\left\{\Delta t_{i j}\right\}$ for $D=0.05$ and 0.06 , respectively; the bin size in each histogram is $2.5 \mathrm{~ms}$. Like the above case, we also consider 5 stages, represented by I (starting from $0 \mathrm{~s}$ ), II (starting from $100 \mathrm{~s}$ ), III (starting from $250 \mathrm{~s}$ ), IV (starting from $500 \mathrm{~s}$ ), and V (starting from $800 \mathrm{~s}$ ). At each stage, we get the distribution of $\left\{\Delta t_{i j}\right\}$ for all synaptic pairs during $0.5 \mathrm{~s}$ and obtain the normalized histogram by dividing the distribution with the total number of synapses. Here, LTD and LTP occur in the black $(\Delta t>0)$ and the gray $(\Delta t<0)$ parts, respectively. For $D=0.05$ (LTD), at the stage I, 9 peaks [the central 1st-order peak and each pair of left and right higher $i$ th-order peaks $(i=2,3,4$, and $5)]$ appear in each histogram of $\left\{\Delta t_{i j}\right\}$, in contrast to the case of full BS where just 3 peaks (the main central peak and a pair of minor left and right peaks) appear [see Fig. 8c1-c6 in Kim and Lim (2018b)]. Due to sparse burstings at every 3 rd or 4 th global cycle of $R_{b}(t)$, nearestneighboring pre- and post-synaptic burst onset times may appear in the following separate stripes in the raster plot of burst onset times, such as the nearest-neighboring, the next-nearest-neighboring, the next-next-nearest-neighboring, and the next-next-next-nearest-neighboring stripes, as well as in the same stripe. As a result, 9 peaks appear in the distribution of $H\left(\Delta t_{i j}\right)$. For the case of stage I, the right black part (LTD) is dominant, in comparison with the left gray part (LTP), and hence the overall net LTD begins to emerge. As the stage is increased, the peaks from the 1 st to the 4th order become intensified (i.e., peaks become narrowed, and then they become sharper), because the amplitude of the 1st peak at $3 T_{G}$ in the IBI histogram is getting dominantly increased. On the other hand, the last 5th-order peaks become very small (see the insets in the stages IV and V where the right 5th-order peak is shown), since the amplitude of the 2nd peak at $4 T_{G}$ in the IBI histogram is getting very small. At the stage $\mathrm{V}$, the effect of LTD in the black part tends to nearly cancel out the effect of LTP in the gray part. For the case of $D=0.06$ (LTP), at the 1st stage, 9 peaks also appear in the histogram of $\left\{\Delta t_{i j}\right\}$, 

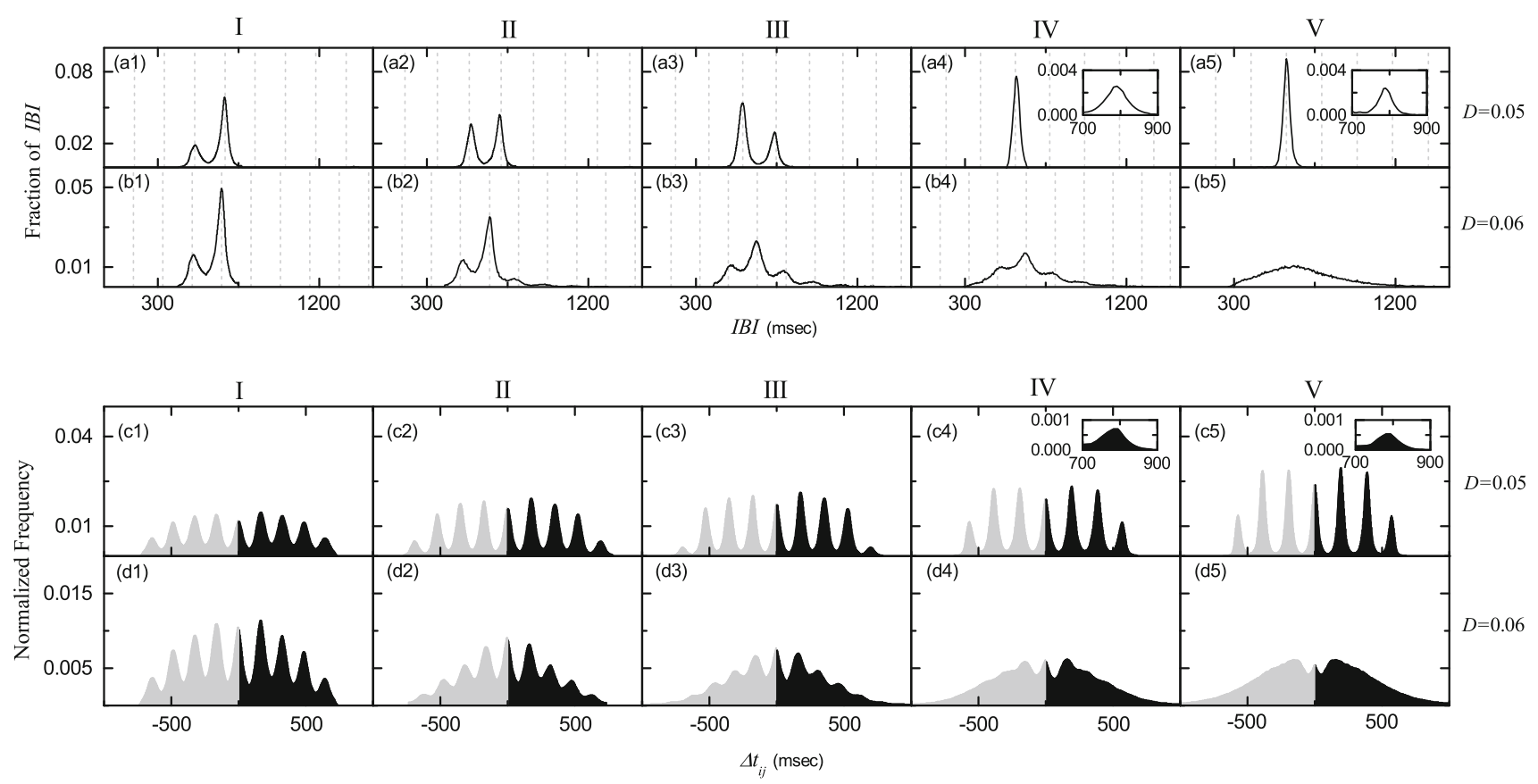

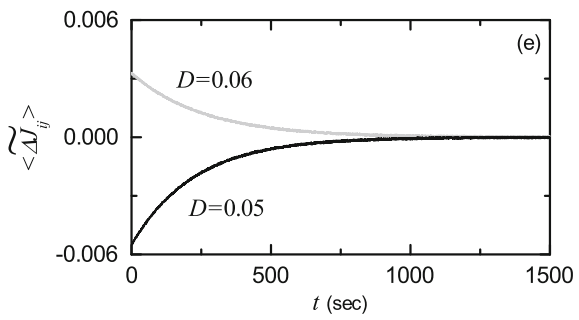

Fig. 7 Microscopic investigations on emergences of LTD and LTP for the case of symmetric attachment with $l^{*}=15 ; N=10^{3}$. Timeevolutions of the IBI histograms for $D=0.05$ in a1-a5 and $D=0.06$ in b1-b5; 5 stages are shown in I (starting from $0 \mathrm{~s}$ ), II (starting from $100 \mathrm{~s}$ ), III (starting from $250 \mathrm{~s}$ ), IV (starting from $500 \mathrm{~s}$ ), and V (starting from $800 \mathrm{~s}$ ). Vertical dotted lines represent multiples of the global period $T_{G}$ of the IPBR $R_{b}(t)$. Time-evolutions of the normalized histogram $H\left(\Delta t_{i j}\right)$ for the distributions of time delays $\left\{\Delta t_{i j}\right\}$ between the pre- and the post-synaptic burst onset times for

as in the case of $D=0.05$. In this case of stage I, the left gray part (LTP) is dominant, in comparison with the right black part (LTD), and hence the overall net LTP begins to occur. However, as the level of stage is increased, peaks become wider, and merging tendency between the peaks is intensified. At the stage $\mathrm{V}$, only one broad central peak seems to appear. At the stage V, the effect of LTP in the gray part tends to nearly cancel out the effect of LTD in the black part.

We now consider successive time intervals $I_{k} \equiv\left(t_{k}, t_{k+1}\right)$, where $t_{k}=0.5 \cdot(k-1) \mathrm{s}(k=1,2,3, \ldots)$. As the time $t$ is increased, we get the $k$ th normalized histogram $H_{k}\left(\Delta t_{i j}\right)(k=1,2,3, \ldots)$ in each $k$ th time interval $I_{k}$, through the distribution of $\left\{\Delta t_{i j}\right\}$ for all synaptic pairs during $0.5 \mathrm{~s}$. Then, from Eq. (7), we get the populationaveraged synaptic strength $\left\langle J_{i j}\right\rangle_{k}$ recursively:

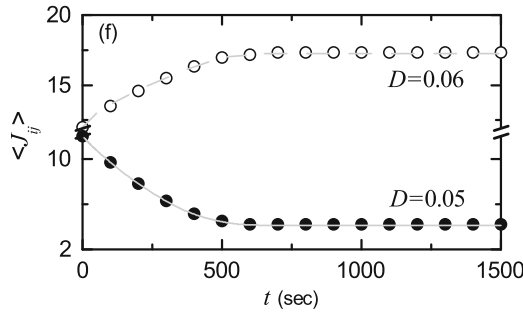

$D=0.05$ in c1-c5 and $D=0.06$ in d1-d5; 5 stages are shown in I (starting from $0 \mathrm{~s}$ ), II (starting from $100 \mathrm{~s}$ ), III (starting from $250 \mathrm{~s}$ ), IV (starting from $500 \mathrm{~s}$ ), and $\mathrm{V}$ (starting from $800 \mathrm{~s}$ ). Timeevolutions of e multiplicative synaptic modification $\left\langle\widetilde{\Delta J_{i j}}\right\rangle$ and $\mathbf{f}$ population-averaged synaptic strength $\left\langle J_{i j}\right\rangle$ (obtained by an approximate method); gray solid and dashed lines represent ones (obtained by direct calculations) for $D=0.05$ and 0.06 in Fig. 5a, respectively

$\left\langle J_{i j}\right\rangle_{k}=\left\langle J_{i j}\right\rangle_{k-1}+\delta \cdot\left\langle\widetilde{\Delta J_{i j}}\left(\Delta t_{i j}\right)\right\rangle_{k}$,

where $\left\langle J_{i j}\right\rangle_{0}=J_{0}(=12)$ and $\langle\cdots\rangle_{k}$ means the average over the distribution of time delays $\left\{\Delta t_{i j}\right\}$ for all synaptic pairs in the $k$ th time interval. Here, the multiplicative synaptic modification $\widetilde{\Delta J_{i j}}\left(\Delta t_{i j}\right)$ is given by the product of the multiplicative factor $\left(J^{*}-J_{i j}\right)\left[J_{i j}\right.$ : synaptic coupling strength at the $(k-1)$ th stage] and the absolute value of synaptic modification $\left|\Delta J_{i j}\left(\Delta t_{i j}\right)\right|$ :

$\widetilde{\Delta J_{i j}}\left(\Delta t_{i j}\right)=\left(J^{*}-J_{i j}\right)\left|\Delta J_{i j}\left(\Delta t_{i j}\right)\right|$.

Then, we get the population-averaged multiplicative synaptic modification $\left\langle\widetilde{\Delta J_{i j}}\left(\Delta t_{i j}\right)\right\rangle_{k}$ at the $k$ th stage through 
a population-average approximation where $J_{i j}$ is replaced by its population average $\left\langle J_{i j}\right\rangle_{k-1}$ at the $(k-1)$ th stage:

$$
\left\langle\widetilde{\Delta J_{i j}}\left(\Delta t_{i j}\right)\right\rangle_{k} \simeq\left(J^{*}-\left\langle J_{i j}\right\rangle_{k-1}\right)\left\langle\left|\Delta J_{i j}\left(\Delta t_{i j}\right)\right|\right\rangle_{k} \text {. }
$$

Here, $\left\langle\left|\Delta J_{i j}\left(\Delta t_{i j}\right)\right|\right\rangle_{k}$ can be easily obtained from the $k$ th normalized histogram $H_{k}\left(\Delta t_{i j}\right)$ :

$$
\left\langle\left|\Delta J_{i j}\left(\Delta t_{i j}\right)\right|\right\rangle_{k} \simeq \sum_{\text {bins }} H_{k}\left(\Delta t_{i j}\right) \cdot\left|\Delta J_{i j}\left(\Delta t_{i j}\right)\right| .
$$

By using Eqs. (16), (18) and (19), we get approximate values of $\left\langle\widetilde{\Delta J_{i j}}\right\rangle_{k}$ and $\left\langle J_{i j}\right\rangle_{k}$ in a recursive way. Figure 7e shows time-evolutions of $\left\langle\widetilde{\Delta J_{i j}}\right\rangle$ for $D=0.05$ (black curve) and $D=0.06$ (gray curve). $\left\langle\widetilde{\Delta J_{i j}}\right\rangle$ for $D=0.05$ is negative. On the other hand, $\left\langle\widetilde{\Delta J_{i j}}\right\rangle$ for $D=0.06$ is positive. For both cases they converge toward nearly zero near the saturation time ( $t=1000 \mathrm{~s}$ ) since the effects of LTD and LTP in the normalized histograms are nearly cancelled out. The timeevolutions of $\left\langle J_{i j}\right\rangle$ for $D=0.05$ (solid circles) and $D=$ 0.06 (open circles) are also given in Fig. 7f. The approximately-obtained values for $\left\langle J_{i j}\right\rangle$ are found to agree well with directly-obtained ones [denoted by the gray solid (dashed) line for $D=0.05(0.06)]$ in Fig. 5a. In this way, LTD (LTP) emerges for $D=0.05(0.06)$.

Finally, in the presence of iSTDP, we investigate the effect of network architecture on BS for $D=0.05$ by varying the symmetric attachment degree $l^{*}$ and the asymmetry parameter $\Delta l$. We first consider the case of symmetric attachment (i.e., $l_{\text {in }}=l_{\text {out }}=l^{*}$ ). Figure 8a shows time-evolutions of population-averaged synaptic strengths $\left\langle J_{i j}\right\rangle$ for various values of $l^{*}$. For each case of $l^{*}=15,23$, and $30,\left\langle J_{i j}\right\rangle$ decreases monotonically below its initial value $J_{0}(=12)$, and it approaches a saturated limit value $\left\langle J_{i j}^{*}\right\rangle$ nearly at $t=1000 \mathrm{~s}$. As a result, LTD occurs for these values of $l^{*}$. On the other hand, for $l^{*}=12\left\langle J_{i j}\right\rangle$ increases monotonically above $J_{0}$, and converges toward a saturated limit value $\left\langle J_{i j}^{*}\right\rangle$. As a result, for this case LTP takes place. Figure $8 \mathrm{~b} 1$ shows a plot of population-averaged limit values of synaptic strengths $\left\langle\left\langle J_{i j}^{*}\right\rangle\right\rangle_{r}$ versus $l^{*}$; the horizontal dotted line represents the initial average value of coupling strengths $J_{0}(=12)$. For $l^{*} \geq 14$ LTD occurs, while for $l^{*} \leq 13$ LTP takes place. Figure $8 \mathrm{~b} 2$ also shows plots of standard deviations $\left\langle\sigma_{J}\right\rangle_{r}$ versus $l^{*}$. With increasing $l^{*},\left\langle\sigma_{J}\right\rangle_{r}$ decreases, but all the values of $\left\langle\sigma_{J}\right\rangle_{r}$ are larger than the initial value $\sigma_{0}(=0.1)$ The LTD (LTP) tends to increase (decrease) the degree of BS due to decrease (increase) in the mean value of synaptic inhibition strengths, and increased standard deviations have a tendency to decrease the degree of BS. We consider the effects of LTD/ LTP on BS after the saturation time $t^{*}(=1000 \mathrm{~s})$. Figure $8 \mathrm{c} 1-\mathrm{c} 4, \mathrm{~d} 1-\mathrm{d} 4$ show raster plots of burst onset times and the corresponding IPBR kernel estimates $R_{b}(t)$ for various values of $l^{*}$, respectively. Due to the dominant effect of LTD (overcoming the effect of increased standard deviation), the degrees of BS for the case of $l^{*}=15,23$, and 30 are increased so much when compared with Fig. 4a3-a5, b3-b5 in the absence of iSTDP. In contrast, for the case of $l^{*}=12$ the population states become desynchronized because of the effects of LTP and increased standard deviation.

We also characterize population behaviors for the BS in terms of the average occupation degree $\left\langle\left\langle O_{i}^{(b)}\right\rangle\right\rangle_{r}$, the average pacing degree $\left\langle\left\langle P_{i}^{(b)}\right\rangle\right\rangle_{r}$, and the statistical-mechanical bursting measure $\left\langle M_{b}\right\rangle_{r}$. Figure 8e1, e2 show plots of $\left\langle\left\langle O_{i}^{(b)}\right\rangle\right\rangle_{r}$ and $\left\langle\left\langle P_{i}^{(b)}\right\rangle\right\rangle_{r}$ (denoted by open circles) versus $l^{*}$, respectively; for comparison, $\left\langle\left\langle O_{i}^{(b)}\right\rangle\right\rangle_{r}$ and $\left\langle\left\langle P_{i}^{(b)}\right\rangle\right\rangle_{r}$ in the absence of iSTDP are also shown in crosses. For $l^{*} \geq 14$, the values of $\left\langle\left\langle O_{i}^{(b)}\right\rangle\right\rangle_{r}$ and $\left\langle\left\langle P_{i}^{(b)}\right\rangle\right\rangle_{r}$ (open circles) are larger than those (crosses) in the absence of iSTDP, because of dominant effect of LTD (overcoming the effect of increased standard deviation). However, in the region of $l^{*} \leq 13$, a rapid transition to desynchronization (i.e. the case of $\left\langle\left\langle P_{i}^{(b)}\right\rangle\right\rangle_{r}=0$ ) occurs due to the effects of LTP and increased standard deviation, in contrast to the smooth decrease in $\left\langle\left\langle P_{i}^{(b)}\right\rangle\right\rangle_{r}$ (crosses) in the absence of iSTDP. The statistical-mechanical bursting measure $\left\langle M_{b}\right\rangle_{r}$ (combining the effect of both the average occupation and pacing degrees) is shown in open circles in Fig. 8e3; for comparison, $\left\langle M_{b}\right\rangle_{r}$ in the absence of iSTDP is also shown in crosses. As in the case in Fig. 6c3, a Matthew effect in inhibitory synaptic plasticity occurs. For $l^{*} \geq 14$, good BS with higher $M_{b}$ gets better because the effect of LTD is dominant in comparison with the effect of increased standard deviation). In contrast, for $l^{*} \leq 13$, bad BS with lower $M_{b}$ gets worse via the effects of both LTP and increased standard deviation. Accordingly, a rapid step-like transition to desynchronization occurs, in contrast to the relatively smooth transition in the absence of iSTDP.

Next, we also consider the case of asymmetric attachment [i.e., $l_{\text {in }}=l^{*}+\Delta l$ and $\left.l_{\text {out }}=l^{*}-\Delta l\left(l^{*}=15\right)\right]$. Time-evolutions of population-averaged synaptic strengths $\left\langle J_{i j}\right\rangle$ for various values of $\Delta l$ are shown in Fig. 8f. In each case of $\Delta l=0,3$, and $6,\left\langle J_{i j}\right\rangle$ decreases monotonically below its initial value $J_{0}(=12)$, and it converges toward a saturated limit value $\left\langle J_{i j}^{*}\right\rangle$ nearly at $t=1000 \mathrm{~s}$. Consequently, LTD occurs for these values of $\Delta l$. In contrast, for $\Delta l=-3,\left\langle J_{i j}\right\rangle$ increases monotonically above $J_{0}$, and approaches a saturated limit value $\left\langle J_{i j}^{*}\right\rangle$. As a result, for this case LTP takes place. A plot of population-averaged limit values of synaptic strengths $\left\langle\left\langle J_{i j}^{*}\right\rangle\right\rangle_{r}$ versus $\Delta l$ is shown in Fig. $8 \mathrm{~g} 1$; the horizontal dotted line represents the initial average value of coupling strengths 

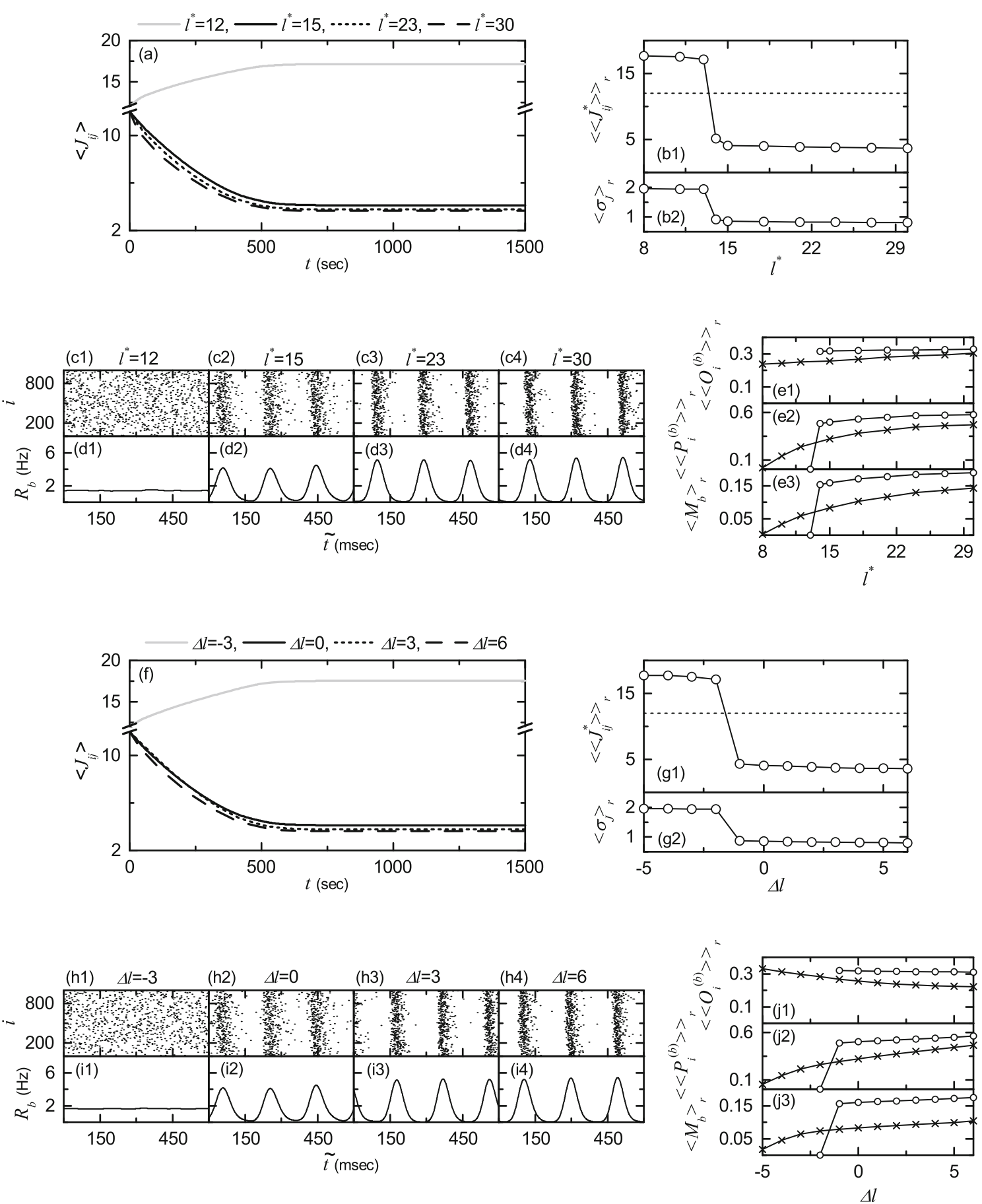

Fig. 8 Effect of network architecture on BS in the presence of iSTDP for $D=0.05 ; \quad N=10^{3}$. Symmetric preferential attachment with $l_{\text {in }}=l_{\text {out }}=l^{*}$. a Time-evolutions of population-averaged synaptic strengths $\left\langle J_{i j}\right\rangle$ for various values of $l^{*}$. Plots of b1 population-averaged limit values of synaptic strengths $\left\langle\left\langle J_{i j}^{*}\right\rangle\right\rangle_{r}\left(J_{i j}^{*}\right.$ : saturated limit values of $J_{i j}$ at $t=1000 \mathrm{~s}$ ) and $\mathbf{b 2}$ standard deviations $\left\langle\sigma_{J}\right\rangle_{r}$ versus $l^{*}$. Raster plots of burst onset times in $\mathbf{c 1 - c 4}$ and IPBR kernel estimates $R_{b}(t)$ in $\mathbf{d 1 - d 4}$ for various values of $l^{*}$ after the saturation time, where $t=t^{*}$ (saturation time: $1000 \mathrm{~s})+\widetilde{t}$. Plots of $\mathbf{e} 1$ the average occupation degree $\left\langle\left\langle O_{i}^{(b)}\right\rangle\right\rangle_{r}$ (open circles), $\mathbf{e} 2$ the average pacing degree $\left\langle\left\langle P_{i}^{(b)}\right\rangle\right\rangle_{r}$ (open circles), and $\mathbf{e} \mathbf{3}$ the statistical-mechanical bursting measure $\left\langle M_{b}\right\rangle_{r}$ (open circles) versus $l^{*}$. For comparison, $\left\langle\left\langle O_{i}^{(b)}\right\rangle\right\rangle_{r},\left\langle\left\langle P_{i}^{(b)}\right\rangle\right\rangle_{r}$, and $\left\langle M_{b}\right\rangle_{r}$ versus $l^{*}$ in the absence of
iSTDP are also denoted by crosses. Asymmetric preferential attachment with $l_{\text {in }}=l^{*}+\Delta l$ and $l_{\text {out }}=l^{*}-\Delta l\left(l^{*}=15\right)$. f Time-evolutions of population-averaged synaptic strengths $\left\langle J_{i j}\right\rangle$ for various values of $\Delta l$. Plots of $\mathbf{g} 1$ population-averaged limit values of synaptic strengths $\left\langle\left\langle J_{i j}^{*}\right\rangle\right\rangle_{r}\left(J_{i j}^{*}\right.$ : saturated limit values of $J_{i j}$ at $t=1000 \mathrm{~s}$ ) and $\mathbf{g} 2$ standard deviations $\left\langle\sigma_{J}\right\rangle_{r}$ versus $\Delta l$. Raster plots of burst onset times in h1-h4 and IPBR kernel estimates $R_{b}(t)$ in $\mathbf{i} \mathbf{1}-\mathbf{i} \mathbf{4}$ for various values of $\Delta l$ after the saturation time, where $t=t^{*}$ (saturation time: $1000 \mathrm{~s}$ ) $+\widetilde{t}$. Plots of $\mathbf{j 1}$ the average occupation degree $\left\langle\left\langle O_{i}^{(b)}\right\rangle\right\rangle_{r}$ (open circles), $\mathbf{j} 2$ the average pacing degree $\left\langle\left\langle P_{i}^{(b)}\right\rangle\right\rangle_{r}$ (open circles), and $\mathbf{j} \mathbf{3}$ the statistical-mechanical bursting measure $\left\langle M_{b}\right\rangle_{r}$ (open circles) versus $\Delta l$. For comparison, $\left\langle\left\langle O_{i}^{(b)}\right\rangle\right\rangle_{r},\left\langle\left\langle P_{i}^{(b)}\right\rangle\right\rangle_{r}$, and $\left\langle M_{b}\right\rangle_{r}$ versus $\Delta l$ in the absence of iSTDP are also denoted by crosses 
$J_{0}(=12)$. For $\Delta l \geq-1$ LTD occurs, while for $\Delta l \leq-2 \mathrm{LTP}$ takes place. Plots of standard deviations $\left\langle\sigma_{J}\right\rangle_{r}$ versus $\Delta l$ are also shown in Fig. 8g2. As $\Delta l$ is increased, $\left\langle\sigma_{J}\right\rangle_{r}$ decreases, but all these values of $\left\langle\sigma_{J}\right\rangle_{r}$ are larger than the initial value $\sigma_{0}(=0.1)$. We also consider the effects of LTD (increasing the degree of BS), LTP (decreasing the degree of BS), and increased standard deviation (decreasing the degree of BS) on $\mathrm{BS}$ after the saturation time $t^{*}(=1000 \mathrm{~s})$. Figure $8 \mathrm{~h} 1-\mathrm{h} 4, \mathrm{i} 1-$ i4 show raster plots of burst onset times and the corresponding IPBR kernel estimates $R_{b}(t)$ for various values of $\Delta l$, respectively. Due to the dominant effect of LTD (overcoming the effect of increased standard deviation) the degrees of BS for the case of $\Delta l=0,3$, and 6 are increased much when compared with Fig. 4e3-e5, f3-f5 in the absence of iSTDP. On the other hand, in the case of $\Delta l=-3$ the population state becomes desynchronized because of the effects of both LTP and increased standard deviation.

We characterize population behaviors for the BS in terms of the average occupation degree $\left\langle\left\langle O_{i}^{(b)}\right\rangle\right\rangle_{r}$, the average pacing degree $\left\langle\left\langle P_{i}^{(b)}\right\rangle\right\rangle_{r}$, and the statistical-mechanical bursting measure $\left\langle M_{b}\right\rangle_{r}$. Plots of $\left\langle\left\langle O_{i}^{(b)}\right\rangle\right\rangle_{r}$ and $\left\langle\left\langle P_{i}^{(b)}\right\rangle\right\rangle_{r}$ (denoted by open circles) versus $\Delta l$ are shown in Fig. $8 \mathrm{j} 1, \mathrm{j} 2$, respectively; for comparison, $\left\langle\left\langle O_{i}^{(b)}\right\rangle\right\rangle_{r}$ and $\left\langle\left\langle P_{i}^{(b)}\right\rangle\right\rangle_{r}$ in the absence of iSTDP are also shown in crosses. For $\Delta l \geq-1$, the values of $\left\langle\left\langle O_{i}^{(b)}\right\rangle\right\rangle_{r}$ and $\left\langle\left\langle P_{i}^{(b)}\right\rangle\right\rangle_{r}$ (open circles) are larger than those (crosses) in the absence of iSTDP, due to the dominant effect of LTD (overcoming the effect of increased standard deviation). However, in the region of $\Delta l \leq-2$, a rapid step-like transition to the case of $\left\langle\left\langle P_{i}^{(b)}\right\rangle\right\rangle_{r}=0$ takes place because of the effects of both LTP and increased standard deviation, in contrast to the smooth decrease in $\left\langle\left\langle P_{i}^{(b)}\right\rangle\right\rangle_{r}$ (crosses) in the absence of iSTDP. Figure $8 \mathrm{j} 3$ shows the statistical-mechanical bursting measure $\left\langle M_{b}\right\rangle_{r}$ (combining the effect of both the average occupation and pacing degrees) in open circles; for comparison, $\left\langle M_{b}\right\rangle_{r}$ in the absence of iSTDP is shown in crosses. Like the above case in Fig. 8e3, a Matthew effect in inhibitory synaptic plasticity occurs. For $\Delta l \geq-1$, good BS with higher $M_{b}$ gets better via the dominant effect of LTD (overcoming the effect of increased standard deviation), while for $\Delta l \leq-2$ bad BS with lower $M_{b}$ gets worse via the effects of LTP and increased standard deviation. Consequently, a rapid transition to desynchronization occurs, in contrast to the relatively smooth transition in the absence of iSTDP.

\section{Summary}

We are concerned about BS, associated with neural information processes in health and disease, in the BarabásiAlbert SFN of inhibitory bursting Hindmarsh-Rose neurons. In previous works on BS, inhibitory synaptic plasticity was not considered (i.e., synaptic inhibition strengths were static). On the other hand, in the present work, adaptive dynamics of synaptic inhibition strengths are governed by the iSTDP. An anti-Hebbian time window has been used for the iSTDP update rule, in contrast to the Hebbian time window for the case of eSTDP. The effects of iSTDP on BS have been investigated by varying the noise intensity $D$ for the case of symmetric preferential attachment with $l^{*}=15$.

Due to inhibition, the roles of LTD (increasing the degree of BS) and LTP (decreasing the degree of BS) for the case of iSTDP are reversed in comparison with those in excitatory synaptic plasticity where the degree of population synchronization is increased (decreased) via LTP (LTD). Increased standard deviations for both cases of LTD and LTP tend to decrease the degree of BS. A Matthew effect has been found in inhibitory synaptic plasticity. In most region of LTD, good BS (with higher bursting measure $M_{b}$ ) gets better due to the dominant effect of LTD (overcoming the effect of increased standard deviation). In contrast, in the region of LTP bad BS (with lower $M_{b}$ ) gets worse because of the effects of both LTP and increased standard deviation. Consequently, near the threshold $D_{t h}$ a rapid transition from $\mathrm{BS}$ to desynchronization occurs via LTP, in contrast to the relatively smooth transition in the absence of iSTDP.

Emergences of LTD and LTP of synaptic inhibition strengths were investigated via a microscopic method based on the distributions of time delays $\left\{\Delta t_{i j}\right\}$ between the nearest burst onset times of the pre- and the post-synaptic neurons. Time evolutions of normalized histograms $H\left(\Delta t_{i j}\right)$ were followed for both cases of LTD and LTP. For the case of LTD with $D=0.05,9$ peaks appear in $H\left(\Delta t_{i j}\right)$ due to sparse BS, in contrast to the case of full BS where only 3 peaks appear. On the other hand, in the case of LTP with $D=0.06$ merging of such multiple peaks occurs. Based on the normalized histogram at each stage, we recursively obtained population-averaged synaptic inhibition strength $\left\langle J_{i j}\right\rangle$ at successive stages by using an approximate recurrence relation. These approximate values of $\left\langle J_{i j}\right\rangle$ were found to agree well with directly-calculated ones. In this way, one can understand clearly how microscopic distributions of $\left\{\Delta t_{i j}\right\}$ contribute to $\left\langle J_{i j}\right\rangle$.

Futhermore, in the presence of iSTDP, we have also studied the effect of network architecture on BS for a fixed value of $D=0.05$ by varying the symmetric attachment degree $l^{*}$ and the asymmetry parameter $\Delta l$. As in the above case of variation in $D$ for $l^{*}=15$, Matthew effects have also been found to occur for both cases of variations in $l^{*}$ and $\Delta l$. For $l^{*} \geq 14$ and $\Delta l \geq-1$, good BS with higher bursting measure $M_{b}$ gets better because the effect of LTD is dominant in comparison with the effect of increased 
standard deviation. On the other hand, for $l^{*} \leq 13$ and $\Delta l \leq-2$, bad BS with lower bursting measure $M_{b}$ gets worse via the effects of both LTP and increased standard deviation.

Finally, we discuss limitations of our present work and future works. In our present work, we consider only a scale-free complex network. As a future work, it would be interesting to study the effect of iSTDP on BS in other networks with different topology (e.g., small-world or allto-all networks). In our previous work (Kim and Lim 2018d), we studied the effect of iSTDP on fast sparse synchronization (FSS) in the small-world neuronal network of inhibitory fast spiking interneurons, and found the same kind of Matthew effect in inhibitory synaptic plasticity; good FSS gets better via LTD, while bad FSS gets worse via LTP. Hence, for our present case of BS, the same kind of Matthew effect in iSTDP is also expected to occur in neuronal networks with different topology. In the real brain, structural synaptic plasticity (i.e. disappearance, appearance, or rewiring of synapses) also occurs (Engert and Bonhoeffer 1999; Butz et al. 2007, 2008, 2014; Caroni et al. 2012; Ganguly and Poo 2013; Gafarov 2016, 2018), in addition to the case of functional synaptic plasticity where only synaptic strengths change without any structural changes. In our present work, we do not consider this kind of structural synaptic plasticity. Hence, the study on effect of structural plasticity on BS would be interesting as a future work. In our work, we consider inhibitory synaptic plasticity in the network consisting of just inhibitory bursting neurons. We note that the iSTDP rule may be applicable to spiking neurons as well as bursting neurons, because a spike may be regarded as a burst with a single spike. Hence, as a future work, it would be interesting to study the effect of iSTDP on population synchronization in the network composed of both spiking and bursting neurons.

Acknowledgements This research was supported by the Basic Science Research Program through the National Research Foundation of Korea (NRF) funded by the Ministry of Education (Grant No. 20162007688).

\section{References}

Abbott LF, Blum KI (1996) Functional significance of long-term potentiation for sequence learning and prediction. Cereb Cortex 6:406-416

Abbott LF, Nelson SB (2000) Synaptic plasticity: taming the beast. Nat Neurosci 3:1178-1183

Albert R, Barabási AL (2002) Statistical mechanics of complex networks. Rev Mod Phys 74:47-97

Barabási AL, Albert R (1999) Emergence of scaling in random networks. Science 286:509-512

Bassett DS, Bullmore E (2006) Small-world brain networks. Neuroscientist $12: 512-523$
Batista CAS, Batista AM, de Pontes JAC, Viana RL, Lopes SR (2007) Chaotic phase synchronization in scale-free networks of bursting neuron. Phys Rev E 76:016218

Batista CAS, Batista AM, de Pontes JAC, Lopes SR, Viana RL (2009) Bursting synchronization in scale-free networks. Chaos Soliton Fract 41:2220-2225

Batista CAS, Lopes SR, Viana RL, Batista AM (2010) Delayed feedback control of bursting synchronization in a scale-free neuronal network. Neural Netw 23:114-124

Batista CA, Lameu EL, Batista AM, Lopes SR, Pereira T, ZamoraLopez G, Kurths J, Viana RL (2012) Phase synchronization of bursting neurons in clustered small-world networks. Phys Rev E 86:016211

Bazhenov M, Timofeev I (2006) Thalamocortical oscillations. Scholarpedia 1(6):1319

Bevan M, Magill P, Terman D, Bolam J, Wilson C (2002) Move to the rhythm: oscillations in the subthalamic nucleus-external globus pallidus network. Trends Neurosci 25:525-531

Bi GQ, Poo MM (1998) Synaptic modifications in cultured hippocampal neurons: dependence on spike timing, synaptic strength, and postsynaptic cell type. J Neurosci 18:10464-10472

Bi GQ, Poo MM (2001) Synaptic modification by correlated activity: Hebb's postulate revisited. Annu Rev Neurosci 24:139-166

Bienenstock EL, Cooper LN, Munro PW (1982) Theory for the development of neuron selectivity: orientation specificity and binocular interaction in visual cortex. J Neurosci 2:32-48

Blum KI, Abbott LF (1996) A model of spatial map formation in the hippocampus of the rat. Neural Comput 8:85-93

Bonifazi P, Goldin M, Picardo MA, Jorquera I, Cattani A, Bianconi G, Represa A, Ben-Ari Y, Cossart R (2009) GABAergic hub neurons orchestrate synchrony in developing hippocampal networks. Science 326:1419-1424

Borges RR, Borges FS, Batista AM, Lameu EL, Viana RL, Iarosz KC, Caldas IL, Viana RL, Sanjuán MAF (2016) Effects of spike timing-dependent plasticity on the synchronization in a random Hodgkin-Huxley neuronal network. Commun Nonlinear Sci Numer Simul 34:12-22

Borges RR, Borges FS, Lameu EL, Batista AM, Iarosz KC, Caldas IL, Antonopoulos CG, Baptista MS (2017a) Spike timing-dependent plasticity induces non-trivial topology in the brain. Neural Netw 88:58-64

Borges RR, Borges FS, Lameu EE, Protachevicz PR, Iarosz KC, Caldas IL, Viana RL, Cacau EEN, Baptista MS, Grebogi C, Batista AM (2017b) Synaptic plasticity and spike synchronisation in neuronal networks. Braz J Phys 47:678-688

Brown P (2007) Abnormal oscillatory synchronisation in the motor system leads to impaired movement. Cur Opin Neurobiol 17:656-664

Brunel N, Wang XJ (2003) What determines the frequency of fast network oscillations with irregular neural discharges? I. Synaptic dynamics and excitation-inhibition balance. J Neurophysiol 90:415-430

Bullmore E, Sporns O (2009) Complex brain networks: graph theoretical analysis of structural and functional systems. Nat Rev Neurosci 10:186-198

Butera RJ, Rinzel J, Smith JC (1999) Models of respiratory rhythm generation in the pre-Botzinger complex. I. Bursting pacemaker neurons. J Neurophysiol 82:382-397

Butz M, Wörgötter F, van Ooyen A (2007) Modelling structural plasticity. BMC Neurosci 2007(8):P194

Butz M, Wörgötter F, van Ooyen A (2008) Activity-dependent structural plasticity. Brain Res Rev 60:287-305

Butz M, Sttenbuck ID, van Ooyen A (2014) Homeostatic structural plasticity increases the efficiency of small-world networks. Front Synaptic Neurosci 6:7 
Buzsáki G, Geisler C, Henze DA, Wang XJ (2004) Interneuron diversity series: circuit complexity and axon wiring economy of cortical interneurons. Trends Neurosci 27:186-193

Caporale N, Dan Y (2008) Spike timing-dependent plasticity: a Hebbian learning rule. Annu Rev Neurosci 31:25-46

Cardin JA (2012) Dissecting local circuits in vivo: integrated optogenetic and electrophysiology approaches for exploring inhibitory regulation of cortical activity. J Physiol (Paris) 106:104-111

Caroni P, Donato F, Muller D (2012) Structural plasticity upon learning: regulation and functions. Nat Rev Neurosci 13:478-490

Castilo PE, Chiu CQ, Carroll RC (2011) Long-term synaptic plasticity at inhibitory synapses. Curr Opin Neurobiol 21:328-338

Chay TR, Keizer J (1983) Minimal model for membrane oscillations in the pancreatic \$ beta \$-cell. Biophys J 42:181-190

Chklovskii DB, Mel BW, Svoboda K (2004) Cortical rewiring and information storage. Nature 431:782-788

Connors BW, Gutnick MJ (1990) Intrinsic firing patterns of diverse neocortical neurons. Trends Neurosci 13:99-104

Coombes S, Bressloff PC (eds) (2005) Bursting: the genesis of rhythm in the nervous system. World Scientific, Singapore

Dan Y, Poo MM (2004) Spike timing-dependent plasticity of neural circuits. Neuron 44:23-30

Dan Y, Poo MM (2006) Spike timing-dependent plasticity: from synapse to perception. Physiol Rev 86:1033-1048

Debanne D, Gähwiler BH, Thompson SM (1998) Long-term synaptic plasticity between pairs of individual CA3 pyramidal cells in rat hippocampal slice cultures. J Physiol 507(1):237-247

Deisseroth K, Feng G, Majewska AK, Miesenbóck G, Ting A, Schnitzer MJ (2006) Next-generation optical technologies for illuminating genetically targeted brain circuits. J Neurosci 26:10380-10386

Del Negro CA, Hsiao CF, Chandler SH, Garfinkel A (1998) Evidence for a novel bursting mechanism in rodent trigeminal neurons. Biophys J 75:174-182

Dhamala M, Jirsa V, Ding M (2004) Transitions to synchrony in coupled bursting neurons. Phys Rev Lett 92:028101

Duan L, Fan D, Lu Q (2013) Hopf bifurcation and bursting synchronization in an excitable systems with chemical delayed coupling. Cogn Neurodyn 7:341-349

Egger V, Feldmeyer D, Sakmann B (1999) Coincidence detection and changes of synaptic efficacy in spiny stellate neurons in rat barrel cortex. Nat Neurosci 2:1098-1105

Eguíluz VM, Chialvo DR, Cecchi GA, Baliki M, Apkarian AV (2005) Scale-free brain functional networks. Phys Rev Lett 94:018102

Elson RC, Selverston AI, Huerta R, Rulkov NF, Rabinovich MI, Abarbanel HDI (1998) Synchronous behavior of two coupled biological neurons. Phys Rev Lett 81:5691-5695

Engert F, Bonhoeffer T (1999) Dendritic spine changes associated with hippocampal long-term synaptic plasticity. Nature 399:66-70

Feldman DE (2000) Timing-based LTP and LTD at vertical inputs to layer II/III pyramidal cells in rat barrel cortex. Neuron 27:45-56

Feldman DE (2012) The spike-timing dependence of plasticity. Neuron 75:556-571

Felleman DJ, Van Essen DC (1991) Distributed hierarchical processing in the primate cerebral cortex. Cereb Cortex 1:1-47

Ferrari FAS, Viana RL, Lopes SR, Stoop R (2015) Phase synchronization of coupled bursting neurons and the generalized Kuramoto model. Neural Netw 66:107-118

Fisher R, van Emde Boas W, Blume W, Elger C, Genton P, Lee P, Engel J (2005) Epileptic seizures and epilepsy: definitions proposed by the International League Against Epilepsy (ILAE) and the International Bureau for Epilepsy (IBE). Epilepsia $46: 470-472$
Freeman LC (1977) A set of measures of centrality based on betweenness. Sociometry 40:35-41

Freeman LC (1978) Centrality in social networks conceptual clarification. Soc Netw 1:215-239

Froemke RC (2015) Plasticity of cortical excitatory-inhibitory balance. Annu Rev Neurosci 38:195-219

Gafarov FM (2016) Emergence of the small-world architecture in neural networks by activity dependent growth. Physica A 461:409-418

Gafarov FM (2018) Neural electrical activity and neural network growth. Neural Netw 101:15-24

Gaiarsa JL, Caillard O, Ben-Ari Y (2002) Long-term plasticity at GABAergic and glycinergic synapses: mechanisms and functional significance. Trends Neurosci 25:564-570

Gais S, Plihal W, Wagner U, Born J (2000) Early sleep triggers memory for early visual discrimination skills. Nat Neurosci 3:1335-1339

Ganguly K, Poo M-M (2013) Activity-dependent neural plasticity from bench to bedside. Neuron 80:729-741

Gerstner W, Kempter R, van Hemmen JL, Wagner H (1996) A neuronal learning rule for sub-millisecond temporal coding. Nature 383:76-81

Grado L, Johnson M, Netoff T (2015) Optimization of deep brain stimulation parameters using computational models of the basal ganglia with spike-time dependent plasticity. Neuroscience 2015(212):05

Gray CM, McCormick DA (1996) Chattering cells: superficial pyramidal neurons contributing to the generation of synchronous oscillations in the visual cortex. Science 274:109-113

Haas J, Nowotny T, Abarbanel H, Zavala B, Landisman C (2006) Spike-timing-dependent plasticity of inhibitory synapses in the entorhinal cortex. J Neurophysiol 96:3305-3313

Hammond C, Bergman H, Brown P (2007) Pathological synchronization in Parkinson's disease: networks, models and treatments. Trends Neurosci 30:357-364

Hebb DO (1949) The organization of behavior; a neuropsychological theory. Wiley, New York

Hindmarsh JL, Rose RM (1982) A model of the nerve impulse using two first-order differential equations. Nature 296:162-164

Hindmarsh JL, Rose RM (1984) A model of neuronal bursting using three coupled first order differential equations. Proc R Soc Lond Ser B 221:87-102

Hrg D (2013) Synchronization of two Hindmarsh-Rose neurons with unidirectional coupling. Neural Netw 40:73-79

Ivanchenko MV, Osipov GV, Shalfeev VD, Kurths J (2004) Phase synchronization in ensembles of bursting oscillators. Phys Rev Lett 93:134101

Izhikevich EM (2000) Neural excitability, spiking and bursting. Int J Bifurc Chaos 10:1171-1266

Izhikevich EM (2004) Which model to use for cortical spiking neurons? IEEE Trans Neural Netw 15:1063-1070

Izhikevich EM (2006) Bursting. Scholarpedia 1(3):1300

Izhikevich EM (2007) Dynamical systems in neuroscience. MIT Press, Cambridge

Izhikevich EM, Desai NS, Walcott EC, Hoppensteadt FC (2003) Bursts as a unit of neural information: selective communication via resonance. Trends Neurosci 26:161-167

Ji D, Wilson M (2007) Coordinated memory replay in the visual cortex and hippocampus during sleep. Nat Neurosci 10:100-107

Jun D, Gaung-jun Z, Yong X, Hong Y, Jeu W (2014) Dynamic behavior analysis of rational-order Hindmarsh-Rose neuronal model. Cogn Neurodyn 8:167-175

Kaiser M, Martin R, Andras P, Young MP (2007) Simulation of robustness against lesions of cortical networks. Eur J Neurosci 25:3185-3192 
Kepecs A, van Rossum MCW, Song S, Tegner J (2002) Spike-timingdependent plasticity: common themes and divergent vistas. Biol Cybern 87:446-458

Kim SY, Lim W (2015a) Noise-induced burst and spike synchronizations in an inhibitory small-world network of subthreshold bursting neurons. Cogn Neurodyn 9:179-200

Kim SY, Lim W (2015b) Thermodynamic order parameters and statistical-mechanical measures for characterization of the burst and spike synchronizations of bursting neurons. Physica A 438:544-559

Kim SY, Lim W (2016) Effect of network architecture on burst and spike synchronization in a scale-free network of bursting neurons. Neural Netw 79:53-77

Kim SY, Lim W (2018a) Stochastic spike synchronization in a smallworld neural network with spike-timing-dependent plasticity. Neural Netw 97:92-106

Kim SY, Lim W (2018b) Effect of spike-timing-dependent plasticity on stochastic burst synchronization in a scale-free neuronal network. Cogn Neurodyn 12:315-342

Kim SY, Lim W (2018c) Effect of spike-timing-dependent plasticity on stochastic spike synchronization in an excitatory neuronal population. In: Delgado-Garcia J, Pan X, Sanchez-Campusano R, Wang R (eds) Advances in Cognitive Neurodynamics (VI). Springer, Singapore, pp 335-341

Kim SY, Lim W (2018d) Effect of inhibitory spike-timing-dependent plasticity on fast sparsely synchronized rhythms in a small-world neuronal network. Neural Netw 106:50-66

Kinard TA, de Vries G, Sherman A, Satin LS (1999) Modulation of the bursting properties of single mouse pancreatic \$beta \$-cells by artificial conductances. Biophys J 76:1423-1435

Kornoski J (1948) Conditional reflexes and neuron organization. Cambridge University Press, Cambridge

Krahe R, Gabbian F (2004) Burst firing in sensory system. Nat Rev Neurosci 5:13-23

Kullmann DM, Moreau AW, Bakiri Y, Nicholson E (2012) Plasticity of Inhibition. Neuron 75:951-962

Lameu EL, Batista CAS, Batista AM, Larosz K, Viana RL, Lopes SR, Kurths J (2012) Suppression of bursting synchronization in clustered scale-free (rich-club) neuronal networks. Chaos 22:043149

Lamsa KP, Kullmann DM, Woodin MA (2010) Spike-timing dependent plasticity in inhibitory circuits. Front Synaptic Neurosci 2:8

Larimer P, Strowbridge BW (2008) Nonrandom local circuits in the dentate gyrus. J Neurosci 28:12212-12223

Lee SH, Govindaiah G, Cox CL (2007) Heterogeneity of firing properties among rat thalamic reticular nucleus neurons. J Physiol 582:195-208

Li X, Ouyang G, Usami A, Ikegaya Y, Sik A (2010) Scale-free topology of the CA3 hippocampal network: a novel method to analyze functional neuronal assemblies. Biophys $\mathbf{J}$ 98:1733-1741

Lisman J (1997) Bursts as a unit of neural information: making unreliable synapse reliable. Trends Neurosci 20:38-43

Llinás RL, Jahnsen H (1982) Electrophysiology of mammalian thalamic neurons in vitro. Nature 297:406-408

Longtin A (1997) Autonomous stochastic resonance in bursting neurons. Phys Rev E 55:868-876

Lourens MAJ, Schwab BC, Nirody JA, Meijer HGE, van Gils SA (2015) Exploiting pallidal plasticity for stimulation in Parkinson's disease. J Neural Eng 12:026005

Markram H, Lübke J, Frotscher M, Sakmann B (1997) Regulation of synaptic efficacy by coincidence of postsynaptic APs and EPSPs. Science 275:213-215
Markram H, Gerstner W, Sjöström PJ (2012) Spike-timing-dependent plasticity: a comprehensive overview. Front Synaptic Neurosci $4: 2$

McCormick DA, Huguenard JR (1992) A Model of the electrophysiological properties of thalamocortical relay neurons. J Neurophysiol 8:1384-1400

Mehta MR, Wilson M (2000) From hippocampus to V1: effect of LTP on spatiotemporal dynamics of receptive fields. Neurocomputing 32:905-911

Meng P, Wang Q, Lu Q (2013) Bursting synchronization dynamics of pancreatic \$lbeta \$-cells with electrical and chemical coupling. Cogn Neurodyn 7:197-212

Merton RK (1968) The Matthew effect in science. Science 159:56-63

Morgan RJ, Soltesz I (2008) Nonrandom connectivity of the epileptic dentate gyrus predicts a major role for neuronal hubs in seizures. Proc Natl Acad Sci U S A 105:6179-6184

Morrison A, Aertsen A, Diesmann M (2007) Spike-timing-dependent plasticity in balanced random networks. Neural Comput 19:1437-1467

Ngouonkadi EBM, Fostin HB, Nono MK, Fotso PHL (2016) Noise effects on robust synchronization of a small pacemaker neuronal ensemble via nonlinear controller: electronic circuit design. Cogn Neurodyn 10:385-404

Nishikawa T, Motter AE, Lai YC, Hoppensteadt FC (2003) Heterogeneity in oscillator networks: are smaller worlds easier to synchronize? Phys Rev Lett 91:014101

Park C, Worth RM, Rubchinsky LL (2010) Fine temporal structure of beta oscillations synchronization in subthalamic nucleus in Parkinson's disease. J Neurophysiol 103:2707-2716

Pereira T, Baptista M, Kurths J (2007) Multi-time-scale synchronization and information processing in bursting neuron networks. Eur Phys J Spec Top 146:155-168

Pernarowski M, Miura RM, Kevorkian J (1992) Perturbation techniques for models of bursting electrical activity in pancreatic \$ Ibeta \$-cells. SIAM J Appl Math 52:1627-1650

Popovych OV, Tass PA (2012) Desynchronizing electrical and sensory coordinated reset neuromodulation. Front Hum Neurosci $6: 58$

Popovych OV, Yanchuk S, Tass PA (2013) Self-organized noise resistance of oscillatory neural networks with spike-timingdependent plasticity. Sci Rep 3:2926

Prado TdeL, Lopes SR, Batista CAS, Kurths J, Viana RL (2014) Synchronization of bursting Hodgkin-Huxley-type neurons in clustered networks. Phys Rev E 90:032818

Rinzel J (1985) Bursting oscillations in an excitable membrane model. In: Sleeman BD, Jarvis RJ (eds) Lecture notes in mathematics, ordinary and partial differential equations, vol 1151. Springer, Berlin, pp 304-316

Rinzel J (1987) A formal classification of bursting mechanisms in excitable systems. In: Teramoto E, Yamaguti M (eds) Lecture notes in biomathematics, mathematical topics in population biology, morphogenesis, and neurosciences, vol 71. Springer, Berlin, pp 267-281

Rose RM, Hindmarsh JL (1985) A model of a thalamic neuron. Proc R Soc Lond Ser B 225:161-193

Rostami Z, Jafari S (2018) Defects formation and spiral waves in a network of neurons in presence of electromagnetic induction. Cogn Neurodyn 12:235-254

Rubin J, Lee DD, Sompolinsky H (2001) Equilibrium properties of temporally asymmetric Hebbian plasticity. Phys Rev Lett 86:364-367

San Miguel M, Toral R (2000) Stochastic effects in physical systems. In: Martinez J, Tiemann R, Tirapegui E (eds) Instabilities and nonequilibrium structures VI. Kluwer Academic Publisher, Dordrecht, pp 35-130 
Scannell JW, Blakemore C, Young MP (1995) Analysis of connectivity in the cat cerebral cortex. J Neurosci 15:1463-1483

Scannell JW, Burns GAPC, Hilgetag CC, O'Neil MA, Young MP (1999) The connectional organization of the cortico-thalamic system of the cat. Cereb Cortex 9:277-299

Sejnowski TJ (1977) Storing covariance with nonlinearly interacting neurons. J Math Biol 4:303-321

Sejnowski TJ, Destexhe A (2000) Why do we sleep? Brain Res 886:208-223

Shatz CJ (1992) The developing brain. Sci Am 267:60-67

Shi X, Lu Q (2005) Firing patterns and complete synchronization of coupled Hindmarsh-Rose neurons. Chin Phys 14:77-85

Shi X, Lu Q (2009) Burst synchronization of electrically and chemically coupled map-based neurons. Physica A 388:2410-2419

Shilnikov A, Cymbalyuk G (2005) Transition between tonic spiking and bursting in a neuron model via the blue-sky catastrophe. Phys Rev Lett 94:048101

Shimazaki H, Shinomoto S (2010) Kernel bandwidth optimization in spike rate estimation. J Comput Neurosci 29:171-182

Song S, Abbott LF (2001) Cortical development and remapping through spike timing-dependent plasticity. Neuron 32:339-350

Song S, Miller KD, Abbott LF (2000) Competitive Hebbian learning through spike-timing-dependent plasticity synaptic plasticity. Nat Neurosci 3:919-926

Song S, Sjöström PJ, Reigl M, Nelson S, Chklovskii DB (2005) Highly nonrandom features of synaptic connectivity in local cortical circuits. PLoS Biol 3:e68

Soto-Trevino C, Thoroughman KA, Marder E, Abbott LF (2001) Activity-dependent modification of inhibitory synapses in models of rhythmic neural networks. Nat Neurosci 4:297-303

Sporns O (2011) Networks of the brain. MIT Press, Cambridge

Sporns O, Honey CJ (2006) Small worlds inside big brains. Proc Natl Acad Sci U S A 103:19219-19220

Sporns O, Tononi G, Edelman GM (2000) Theoretical neuroanatomy: relating anatomical and functional connectivity in graphs and cortical connection matrices. Cereb Cortex 10:127-141

Sporns O, Chialvo DR, Kaiser M, Hilgetag CC (2004) Organization, development and function of complex brain networks. Trends Cogn Sci 8:418-425

Stent GS (1973) A physiological mechanism for Hebb's postulate of learning. Proc Natl Acad Sci U S A 70:997-1001

Steriade M, McCormick DA, Sejnowski TJ (1993) Thalamocortical oscillations in the sleeping and aroused brain. Science 262:679-685

Stern EA, Jaeger D, Wilson CJ (1998) Membrane potential synchrony of simultaneously recorded striatal spiny neurons in vivo. Nature 394:475-478

Su H, Alroy G, Kirson ED, Yaari Y (2001) Extracellular calcium modulates persistent sodium current-dependent burst-firing in hippocampal pyramidal neurons. J Neurosci 21:4173-4182

Sun X, Lei J, Perc M, Kurths J, Chen G (2011) Burst synchronization transitions in a neuronal network of subnetworks. Chaos 21:016110

Talathi SS, Hwang DU, Ditto WL (2008) Spike timing dependent plasticity promotes synchrony of inhibitory networks in the presence of heterogeneity. J Comput Neurosci 25:262-281

Tanaka G, Ibarz B, Sanjuan MA, Aihara K (2006) Synchronization and propagation of bursts in networks of coupled map neurons. Chaos 16:013113
Tzounopoulos T, Kim Y, Oertel D, Trussell LO (2004) Cell-specific, spike timing-dependent plasticities in the dorsal cochlear nucleus. Nat Neurosci 7:719-725

Uhlhaas PJ, Singer W (2006) Neural synchrony in brain disorders: relevance for cognitive dysfunctions and pathophysiology. Neuron 52:155-168

van Vreeswijk C, Hansel D (2001) Patterns of synchrony in neural networks with spike adaptation. Neural Comput 13:959-992

Varona P, Torres JJ, Abarbanel HDI, Rabinovych MI, Elson RC (2001) Dynamics of two electrically coupled chaotic neurons: experimental observations and model analysis. Biol Cybern 84:91-101

Vogels TP, Sprekeler H, Zenke F, Clopath C, Gerstner W (2011) Inhibitory plasticity balances excitation and inhibition in sensory pathways and memory networks. Science 334:1569-1573

Vogels TP, Froemke RC, Doyon N, Gilson M, Haas JS, Liu R, Maffei A, Miller P, Wierenga CJ, Wooding MA, Zenke F, Sprekeler H (2013) Inhibitory synaptic plasticity: spike timing-dependence and putative network function. Front Neural Circuits 7:119

von der Malsburg C (1973) Self-organization of orientation sensitive cells in the striate cortex. Kybernetik 14:85-100

Wang Q, Perc M, Duan Z, Chen G (2009) Synchronization transitions on scale-free neuronal networks due to finite information transmission delays. Phys Rev E 80:026206

Wang QY, Murks A, Perc M, Lu QS (2011a) Taming desynchronized bursting with delays in the Macaque cortical network. Chin Phys B 20:040504

Wang Q, Chen G, Perc M (2011b) Synchronous bursts on scale-free neuronal networks with attractive and repulsive coupling. PLoS One 6:e15851

Wang H, Wang Q, Lu Q, Zheng Y (2013) Equilibrium analysis and phase synchronization of two coupled HR neurons with gap junction. Cogn Neurodyn 7:121-131

Wiedemann C (2010) Neuronal networks: a hub of activity. Nat Rev Neurosci 11:74

Wittenberg GM, Wang SS (2006) Malleability of spike-timingdependent plasticity at the CA3-CA1 synapse. J Neurosci 26:6610-6617

Womack MD, Khodakhah K (2002) Active contribution of dendrites to the tonic and trimodal patterns of activity in cerebellar Purkinje neurons. J Neurosci 22:10603-10612

Woodin MA, Ganguly K, Poo MM (2003) Coincident pre- and postsynaptic activity modifies GABAergic synapses by postsynaptic changes in Cl-transporter activity. Neuron 39:807-820

Young MP (1993) The organization of neural systems in the primate cerebral cortex. Philos Trans R Soc 252:13-18

Young MP, Scannell JW, Burns GA, Blakemore C (1994) Analysis of connectivity: neural systems in the cerebral cortex. Rev Neurosci 5:227-250

Yu H, Wang J, Deng B, Wei X, Wong YK, Chan WL, Tsang KM, Yu Z (2011) Chaotic phase synchronization in small-world networks of bursting neurons. Chaos 21:013127

Zhang LI, Tao HW, Holt CE, Harris WA, Poo M (1998) A critical window for cooperation and competition among developing retinorectal synapses. Nature 395:37-44

Zhu J, Liu X (2018) Measuring spike timing distance in the Hindmarsh-Rose neurons. Cogn Neurodyn 12:225-234 